Efetividade de um programa de treino de equilíbrio no estado funcional e na frequência de quedas em mulheres idosas com osteoporose: estudo randomizado e controlado

Dissertação apresentada à Faculdade de Medicina da Universidade de São Paulo para obtenção do título de Mestre em Ciências

Área de Concentração: Reumatologia

Orientadora: Profa. Dra. Rosa Maria Rodrigues Pereira

São Paulo

2006 


\section{Dados Internacionais de Catalogação na Publicação (CIP)}

Preparada pela Biblioteca da

Faculdade de Medicina da Universidade de São Paulo

Creprodução autorizada pelo autor

Madureira, Melisa Moreira

Efetividade de um programa de treino de equilíbrio no estado funcional e na freqüência de quedas em mulheres idosas com osteoporose : estudo randomizado e controlado / Melisa Moreira Madureira. -- São Paulo, 2006.

Dissertação(mestrado)--Faculdade de Medicina da Universidade de São Paulo.

Departamento de Clínica Médica.

Área de concentração: Reumatologia.

Orientadora: Rosa Maria Rodrigues Pereira.

Descritores: 1.Acidentes por quedas/prevenção \& controle

2.Osteoporose/reabilitação 3.Equilíbrio músculo-esquelético 4.Limitação de mobilidade 5.Exercício 6.Aptidão física 7.Idoso 8.Ensaios clínicos controlados aleatórios

USP/FM/SBD-347/06 
"Deus não escolhe os capacitados, mas capacita os escolhidos".

(Anônimo) 


\section{DEDICATÓRIA}

A Deus pelo dom da vida e por todas as oportunidades oferecidas para a realização deste sonho. Por me fazer acreditar que, para vivê-lo, é importante sonhar e buscar com determinação os objetivos almejados.

Aos meus pais, Jonas e Neuza que em todo o meu viver sempre me encorajaram a perseverar, proporcionando-me bases sólidas. Agradeço pelas boas coisas que me desejam por tudo o que sonharam e por acreditarem em meu potencial e pelo constante apoio em todas as etapas de minha vida.

Aos meus irmãos, Jonas e Mariú, pela alegria e efetiva presença em todos os momentos, pelo amor, pela amizade e torcida eterna.

Dizem que amigos são irmãos que escolhemos. Cristiano e Juliana são algumas dessas pessoas que eu escolheria para serem meus irmãos. Agradeço por tê-los conhecido, pelas horas alegres e tristes, pela amizade sincera.

A todos os meus familiares, Moreiras e Madureiras, pelos votos de sucesso e ensinamentos passados de geração a geração.

A minha querida sobrinha Heloísa, motivo de grande alegria em toda família. 


\section{AGRADECIMENTOS}

A Profa. Dra. Rosa Maria Rodrigues Pereira, médica assistente da Disciplina de Reumatologia da FMUSP, minha querida orientadora, por representar o meu espelho da ciência; pelo exemplo de competência e profissionalismo; por depositar plena confiança em meu desenvolvimento científico-profissional e, sobretudo, pela amizade sincera no decorrer desses anos.

À Profa. Dra. Eloisa Dutra de Oliveira Bonfá, professora titular e chefe da Disciplina de Reumatologia, por abrir as portas de pesquisa da Disciplina de Reumatologia da FMUSP e deixá-las abertas permitindo a realização deste trabalho.

À Liliam Takayama, pela inestimável amizade, companhia e pela ajuda na tabulação de dados e organização da pasta do protocolo.

À Valéria de Falco Caparbo, pela amizade, pela disponibilidade e pelo auxílio no serviço do Laboratório de Doenças Osteometabólicas da FMUSP.

Às fisioterapeutas Andréa Lopes Gallinaro e Renata Alqualo Costa, por concederem os alunos do quarto ano de fisioterapia da UNICID para realização da parte prática deste estudo. 
À Cláudia, Fátima, Marta e Iná, pela disponibilidade, pronto interesse e dedicação frente à resolução dos freqüentes problemas levados por mim junto ao HCFMUSP e à pós-graduação.

À Associação Atlética Acadêmica Oswaldo Cruz (AAAOC), por ceder todo o espaço físico para a realização dos exercícios.

Ao Centro de Estudos em Reumatologia de São Paulo, pelo apoio financeiro.

À Merck Sharp \& Dohme, pela impressão e encadernação desta tese e da cartilha de orientação para as pacientes.

À CAPES, pelo auxílio financeiro fornecido ao presente trabalho, pela bolsa de pesquisador e, principalmente, pela grande contribuição à construção da pesquisa em nosso país.

Às pacientes do Grupo de Osteoporose, por serem o motivo principal de todo este trabalho e por me proporcionar um grande prazer em exercer a minha profissão. Esta é uma tentativa de proporcionar-lhes essencialmente melhor qualidade de vida. 
Esta dissertação está de acordo com:

Referências: adaptado de International Committee of Medical Journals Editors (Vancouver)

Universidade de São Paulo. Faculdade de medicina. Serviço de Biblioteca e Documentação.

Guia de apresentação de dissertações, teses e monografias. Elaborado por Anneliese Carneiro da Cunha, Maria Júlia de A. L. Freddi, Maria F. Crestana, Marinalva de Souza Aragão, Suely Campos Cardoso, Valéria Vilhena. São Paulo: Serviço de Biblioteca e Documentação; 2004.

Abreviaturas dos títulos dos periódicos de acordo com List of Journals Indexed in Index Medicus. 


\section{SUMÁRIO}

Resumo

Summary

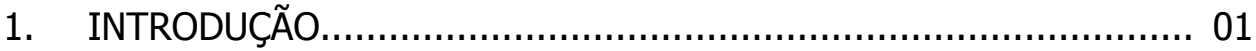

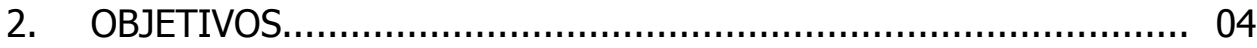

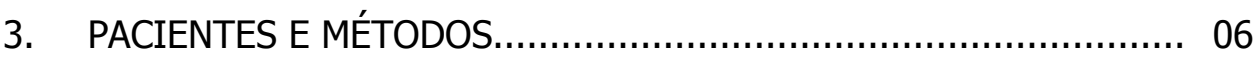

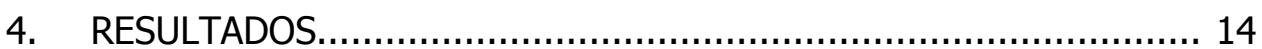

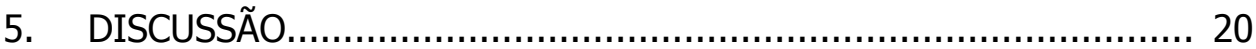

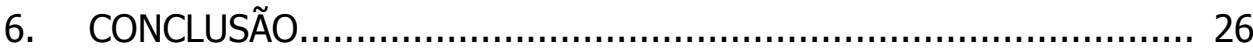

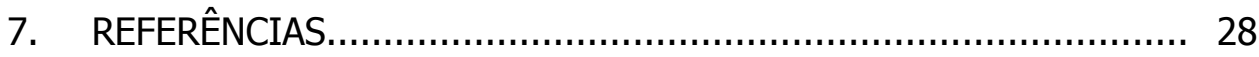

8. ANEXOS ....................................................................... 35

ANEXO 1. Aprovação da Comissão de Ética................................ 36

ANEXO 2. Termo de consentimento livre e esclarecido................. 37

ANEXO 3. Ficha de coleta de dados....................................... 42

ANEXO 4. Ficha de avaliação do estado funcional......................... 45

ANEXO 5. Cartilha de orientação à atividade física ...................... 50

ANEXO 6. Carta de aceite do artigo enviado para o periódico:

Osteoporosis International................................... 76

ANEXO 7. Artigo original em inglês enviado para publicação......... 77 


\section{RESUMO}

Madureira MM. Efetividade de um programa de treino de equilíbrio no estado funcional e na freqüência de quedas em mulheres idosas com osteoporose: estudo randomizado e controlado [dissertação]. São Paulo: Faculdade de Medicina, Universidade de são Paulo; 2007. 84p.

INTRODUÇÃO: O propósito deste estudo foi investigar a efetividade de um programa de treino de equilíbrio na mobilidade, no equilíbrio funcional e estático, e nas quedas em mulheres com osteoporose. MÉTODOS: foram selecionadas consecutivamente 66 mulheres idosas do Ambulatório de Doenças Osteometabólicas e randomizadas em 2 grupos: Intervenção composto de 34 pacientes submetidas ao treino de equilíbrio, e Controle composto de 32 pacientes sem intervenção. O equilíbrio funcional e estático, a mobilidade e as quedas foram avaliados antes e após 12 meses da intervenção utilizando-se: Berg Balance Scale (BBS), Clinical Test Sensory Interaction Balance (CTSIB) e Timed "Up and Go" Test (TUGT). A intervenção utilizou técnicas para melhorar o equilíbrio e a mobilidade, consistindo de 1 hora sessão/semana, e um programa de exercícios em casa. RESULTADOS: Sessenta mulheres completaram o estudo e foram analisadas. Uma diferença no escore da BBS foi significativamente maior no grupo Intervenção comparada ao Controle $(5,5 \pm 5,67$ vs. $-0,5 \pm 4,88$ e $P<0,001)$. Semelhantemente, o número de pacientes no grupo Intervenção apresentou 
melhora em duas condições do CTSIB comparado com o Controle (condição 5 - olhos vendados e superfície instável: 13 vs. 1 paciente, $P<0,001$; e condição 6 - olhos abertos, superfície instável e cúpula visual 12 vs. 1 paciente, $P=0,001)$. Adicionalmente, as diferenças entre o TU\&GT foram reduzidas no grupo Intervenção comparado com o Controle $(-3,65 \pm 3,61$ vs. $2,27 \pm 7,18$ segundos, $\mathrm{P}<0,001)$. Notavelmente, essa melhora foi paralela à redução do número de quedas/paciente no grupo Intervenção comparada ao Controle $(-0,77 \pm 1,76$ vs. $0,33 \pm 0,96, P=0,018)$. CONCLUSÃO: este estudo prospectivo longitudinal demonstrou que uma intervenção utilizando o treino de equilíbrio é extremamente efetiva na melhora do equilíbrio funcional e estático, da mobilidade e redução das quedas em mulheres idosas com osteoporose.

Descritores: 1.Acidentes por quedas/prevenção \& controle; 2.Osteoporose/reabilitação; 3.Equilíbrio músculo-esquelético; 4.Limitação de mobilidade; 5.Exercício; 6.Aptidão física; 7.Idoso; 8.Ensaios clínicos controlados aleatórios. 


\section{SUMMARY}

Madureira MM. Effectiveness of a balance training program on functional status and falling frequency in elderly women with osteoporosis: a randomized controlled study. [dissertation]. São Paulo: "Faculdade de Medicina, Universidade de São Paulo"; 2007. 84p.

INTRODUCTION: The purpose of this study was to investigate the effect of a 12-month Balance Training Program on balance, mobility and falling frequency in women with osteoporosis. METHODS: Sixty-six consecutive elderly women were selected from the Osteometabolic Disease Outpatient Clinic and randomized into 2 groups: the Intervention Group, submitted for balance training; and the Control, without intervention. Balance, mobility and falling frequency were evaluated before and at the end of the trial using: the Berg Balance Scale (BBS), the Clinical Test Sensory Interaction Balance (CTSIB) and the Timed "Up \& Go" Test (TUGT). Intervention used techniques to improve balance consisting of one hour sessions/week and a home-based exercise program. RESULTS: Sixty women completed the study and were analyzed. The BBS difference was significant higher in the intervention compared to control $(5.5 \pm 5.67$ vs. $-0.5 \pm 4.88$ score, $\mathrm{P}<0.001)$. Similarly, the number of patients in intervention group presented improvement in 2 conditions of CTSIB compared to controls (eyes closed and compliance surface condition: 13 vs. 1 patient, $\mathrm{P}<0.001$; eyes open, visual 
conflict and compliance condition: 12 vs. 1 patient, $\mathrm{P}=0.001)$. Additionally, the difference between the TUGT were reduced in the intervention group compared to control $(-3.65 \pm 3.61$ vs. $2.27 \pm 7.18$ seconds, $\mathrm{P}<0.001)$. Notably, this improvement was paralleled by a reduction in the number of falls in the intervention group compared to control $(-0.77 \pm 1.76$ vs. $0.33 \pm 0.96, \mathrm{P}<0.018)$. CONCLUSION: This longitudinal prospective study demonstrated that a intervention using balance training is effective in improving functional and static balance, mobility and falling frequency in elderly women with osteoporosis.

Descriptors: 1.Accidents by falls/prevention \& control; 2.Osteoporosis/rehabilitation; 3.Musculoskeletal balance; 4.Mobility limitation; 5.Exercise; 6.Physical aptitude; 7.Elderly; 8.Controlled random clinical trials. 
INTRODUÇÃO 
A osteoporose é uma doença sistêmica debilitante, que afeta aproximadamente $55 \%$ da população acima dos 50 anos nos E.U.A. ${ }^{1}$. As quedas entre idosos, especialmente com osteoporose, estão associadas à alta morbidade e mortalidade e podem envolver um alto custo nas intervenções médicas ${ }^{2}$. De fato, as quedas são responsáveis por $90 \%$ do crescente aumento das fraturas de quadril ${ }^{3}$ e são a sexta causa de morte em pacientes com mais de 65 anos $^{4}$.

A maioria das quedas envolve múltiplos fatores e as causas de queda são categorizadas em fatores intrínsecos (pessoal) e extrínsecos (ambiental) ${ }^{5,6}$. Alguns exemplos de fatores intrínsecos incluem: doenças neurológicas, doenças músculo-esqueléticas, deterioração sensorial, hipotensão postural, equilíbrio alterado e uso de medicação .

Pesquisas mostram que o equilíbrio alterado é o maior colaborador para quedas em idosos ${ }^{6,8-12}$, existindo uma alta correlação entre déficit do equilíbrio e incidência de quedas ${ }^{13,14}$.

Por essa razão, o estudo do risco de quedas na osteoporose é de extrema importância na intervenção clínica. Reduzir a incidência de quedas é uma prioridade de saúde, refletindo na qualidade de vida e nos custos de saúde ${ }^{2}$.

Além disso, aumenta-se a evidência de que exercícios reduzem o risco de fraturas, por terem um efeito na manutenção da massa óssea e, ainda mais importante por melhorarem a estabilidade postural, a mobilidade e, conseqüentemente, proporcionarem a redução no risco de quedas ${ }^{15}$. 
Certamente, a melhora do equilíbrio deve ser um objetivo na prevenção de quedas ${ }^{16}$.

No entanto, o conhecimento da relação entre o déficit de equilíbrio e a probabilidade de quedas ainda é limitado ${ }^{17}$. A literatura mostra que 0 exercício pode ou não ser eficiente no controle das quedas ${ }^{18}$, assim como o impacto de programas de prevenção com treino de equilíbrio na redução de quedas ainda não está bem estabelecido, principalmente em mulheres com osteoporose.

O treino de equilíbrio tem sido investigado em idosos saudáveis ${ }^{3,16,19-21}$; porém existe somente um estudo do treino de equilíbrio em mulheres com osteoporose. Uma vez que pacientes com osteoporose são particularmente de alto risco para fraturas decorrentes de quedas, maiores pesquisas com este grupo devem ser priorizadas ${ }^{22}$. 
O propósito deste estudo foi investigar a efetividade de um programa de treino de equilíbrio, realizado durante 12 meses em mulheres idosas com osteoporose:

- no equilíbrio funcional,

- no equilíbrio estático,

- na mobilidade funcional e

- na redução de quedas. 
Sessenta e seis mulheres idosas com mais de 65 anos foram selecionadas, consecutivamente, do Ambulatório de Doenças Osteometabólicas do Departamento de Reumatologia da Faculdade de Medicina da Universidade de São Paulo (FMUSP). Somente pacientes com diagnóstico de osteoporose, classificadas de acordo com a Organização Mundial de Saúde ${ }^{23}$, foram incluídas, isto é, com densidade mineral óssea (DMO) abaixo de $-2,5$ desvios-padrão em relação a adultos jovens, na região de coluna lombar ou colo de fêmur ou fêmur total.

Foram excluídas: mulheres com osteoporose secundária, com deficiência visual e auditiva grave, com alteração vestibular de quadro clínico importante; mulheres que utilizassem dispositivo de auxílio à marcha; mulheres que não fossem capazes de caminhar, independentemente, menos que 10 metros, que praticassem exercícios moderados e intensos mais que 8 horas por semana ${ }^{24}$, que planejassem se ausentar da cidade por mais de 4 semanas durante os 12 meses do estudo e mulheres que apresentassem contra-indicações absolutas ou relativas para o treino de exercício de acordo com o Colégio Americano de Medicina do Esporte ${ }^{25}$.

Todas as pacientes recebiam o mesmo tratamento medicamentoso para osteoporose padronizado no ambulatório.

As pacientes foram randomizadas consecutivamente em dois grupos: o grupo submetido ao Programa de Treino de Equilíbrio (Grupo Intervenção GI) - consistindo de 34 pacientes e o Grupo Controle (GC), consistindo de 32 pacientes, sem intervenção. O grupo controle recebia somente o tratamento 
para osteoporose e a orientação para prevenção de quedas e retornavam regularmente ao Ambulatório de Doenças Osteometabólicas. Todas as pacientes leram e assinaram o termo de consentimento que descrevia os procedimentos a serem realizados durante a pesquisa.

\section{Entrevista e registros do prontuário médico}

Através da entrevista e do prontuário médico, foram avaliados dados pessoais, familiares e clínicos, com ênfase em relação à história de fraturas, ao número de quedas no último ano, ao uso de medicamentos para osteoporose e às medicações que favorecem o risco de quedas (hipnóticos, hipotensores, antidepressivos).

\section{Avaliação do estado funcional}

O equilíbrio estático e dinâmico e a mobilidade foram avaliados em ambos os grupos (GI e GC) no início e após 12 meses do programa.

\section{Equilíbrio funcional}

Utilizou-se a Escala de Equilíbrio de Berg (Berg Balance Scale - BBS) ${ }^{26}$, versão brasileira ${ }^{28}$, para avaliar o desempenho do equilíbrio funcional. Essa escala é baseada em 14 itens comuns das atividades de vida diária. O escore máximo que pode ser alcançado é 56 e cada item possui uma escala ordinal de cinco alternativas que variam de 0 a 4 pontos. 
O teste é simples, fácil de aplicar e fazer o acompanhamento da evolução do equilíbrio em pacientes idosos. Requer somente uma régua e um relógio com duração de aproximadamente 15 minutos para ser executado $^{26}$. Escore menor ou igual que 45 é considerado equilíbrio alterado $^{27}$.

\section{Equilíbrio estático}

O equilíbrio estático foi avaliado pelo Teste Clínico de Interação Sensorial no Equilíbrio (Clinical Test of Sensory Interaction for Balance CTSIB) que consiste em seis condições sensoriais: três tarefas e duas diferentes bases de sustentação. $\mathrm{O}$ equilíbrio é considerado alterado se o indivíduo não conseguir permanecer pelo menos 30 segundos nas seis condições $^{28}$.

A melhora do teste foi definida se o paciente completasse o teste no final da avaliação não tendo sido capaz de realizá-lo na primeira avaliação.

\section{Mobilidade funcional}

A mobilidade funcional foi avaliada pelo Teste do Tempo de Ir e Vir (Timed "Up \& Go" Test - TUGT) ${ }^{29}$ que conta o tempo que uma pessoa leva para levantar-se de uma cadeira, caminhar três metros, dar uma volta, andar de volta e sentar-se novamente na cadeira.

Idosos sem déficit de equilíbrio são capazes de completar o teste em menos de 10 segundos. 


\section{Quedas}

O número de quedas, no ano anterior, foi questionado e anotado na primeira avaliação e no final do estudo $(12 \text { meses })^{30}$. Durante o ano do estudo, ambos os grupos recebiam um diário e eram orientados a escrever o dia em que caíram.

Ao final do estudo, a diferença no número de quedas/paciente (avaliação final - avaliação inicial) foi comparada entre o Grupo Intervenção e Controle.

\section{Intervenção}

O Programa de treino de equilíbrio consistiu de 1 hora de exercícios realizados uma vez por semana (40 sessões no total), com supervisão de um fisioterapeuta experiente. Esse programa foi realizado em um clube pertencente ao Hospital das Clinicas da FMUSP e próximo a ele (Associação Atlética Acadêmica Oswaldo Cruz - AAAOC).

Foram utilizados os exercícios de equilíbrio descritos por Tinetti e Suzuki ${ }^{3,11}$, sendo contra-indicados os exercícios de flexão e rotação da coluna. Foram realizados 13 exercícios ativo-livres compostos de uma série com 10 repetições. O tempo para cada alongamento foi de 30 segundos e 0 tempo para cada exercício de equilíbrio foi de 5 minutos.

A intensidade utilizada foi de leve à moderada, os exercícios utilizados no presente estudo foram escolhidos de acordo com a literatura ${ }^{3}$, pois estes poderiam ser realizados por idosos em casa. 
No início do estudo, o grupo equilíbrio foi dividido em dois dias da semana até o aprendizado de todos os exercícios. Após a compreensão, uniu-se o grupo em somente um dia da semana. Uma lista de presença semanal controlou as faltas de cada paciente.

\section{Exercícios básicos de aquecimento e alongamento}

Antes do treinamento, as pacientes participavam de 15 minutos de exercícios de alongamento e aquecimento, consistindo de rotação de cabeça, rotação de ombro, alongamento para membros superiores e inferiores. A caminhada foi feita durante 15 minutos com a supervisão de um fisioterapeuta que, durante a marcha, associava exercícios para membros superiores.

\section{Treino de equilíbrio}

O treino de equilíbrio foi realizado nas posições dinâmica e estática com duração de 30 minutos. O mesmo consistia de: marchar na posição de Tandem (um pé na frente do outro), andar na ponta dos pés e sob calcanhar, andar de lado, andar levantando a perna e o braço contra-lateral. As posições estáticas também foram estimuladas: ficar em um pé só e ficar em posição de Tandem aumentando gradativamente 0 tempo de permanência nestas tarefas ${ }^{3,31}$. 


\section{Exercícios em casa}

As pacientes foram instruídas e incentivadas a dar continuidade aos mesmos exercícios em casa, pelo menos três vezes por semana, durante 30 minutos. Uma cartilha com instruções e ilustrações de cada exercício foi dada a elas. A freqüência da participação dos exercícios em casa foi registrada toda semana pelo fisioterapeuta em uma planilha.

A cartilha era composta de orientações sobre: osteoporose (fatores de risco, diagnóstico e prevenção); exercícios para treinar o equilíbrio; exercícios de alongamento; exercícios para fortalecimento muscular; exercícios contra-indicados na osteoporose; dicas de postura (proteção articular e conservação de energia); prevenção de quedas e orientação alimentar.

\section{Análise estatística}

Sessenta e seis pacientes foram randomizadas em nosso estudo. A análise de dados foi feita em 60 pacientes (30 Grupo Intervenção e 30 Grupo Controle), uma vez que seis delas desistiram (quatro do Grupo Intervenção e duas do Grupo Controle).

No Grupo Equilíbrio, as razões de desistência foram: limitações físicas decorrentes de quedas $(n=1)$, dor nos pés $(n=1)$, motivos pessoais $(n=$ 2); enquanto que, no Grupo Controle, os motivos foram: limitações físicas decorrentes de quedas $(n=1)$ e motivos pessoais $(n=1)$. 
Os dados são apresentados como média e desvios-padrão (DP) para cada variável. As diferenças entre os grupos equilíbrio e controle foram testadas pelo teste t-Student ou Mann-Whitney.

O teste qui-quadrado ou exato de Fisher foi utilizado para comparar o número de pacientes em ambos os grupos (Grupo Intervenção vs. Controle) para uso de medicações, história de fratura e as seis condições sensoriais do CTSIB.

Valores de $\mathrm{P}<0,05$ foram considerados significantes. 
RESULTADOS 
As características dos pacientes, na entrada do estudo, foram similares nos dois grupos em relação à idade: índice de massa corpórea (IMC), história de fraturas, tratamento da osteoporose, uso de diuréticos, hipnóticos/antidepressivos e densidade mineral óssea (DMO), não havendo diferenças estatisticamente significantes entre os dois grupos (Tabela I).

De forma semelhante, na primeira avaliação, não houve diferença, comparando os Grupos Intervenção e Controle, referente ao escore da Escala de Berg (BBS); número de pacientes que não completaram o Teste Cínico para Avaliação da Integração Sensorial no Equilíbrio (CTSIB) nas seis condições sensoriais; Teste do Tempo de Ir e Vir (TUGT) e número de quedas/paciente no último ano no grupo Intervenção e Controle $(P>0,05)$ (Tabela II). 
TABELA I. Dados no início do estudo em relação aos parâmetros antropométricos, história de fraturas, uso de medicações e valores de densidade mineral óssea (T-score) nos Grupos Intervenção e Controle.

\begin{tabular}{|c|c|c|c|}
\hline Variáveis & $\begin{array}{l}\text { Intervenção } \\
\qquad(n=30)\end{array}$ & $\begin{array}{l}\text { Controle } \\
(n=30)\end{array}$ & $P$ \\
\hline Idade, anos & $74,57 \pm 4,82$ & $73,40 \pm 4,61$ & $0,342 *$ \\
\hline IMC, $\mathrm{kg} / \mathrm{m}^{2}$ & $24,39 \pm 4,49$ & $26,51 \pm 5,32$ & $0,100 *$ \\
\hline História de fraturas, \% & $13(43,33)$ & $16(53,3)$ & $0,438^{\&}$ \\
\hline Medicamentos usados para OP, $\mathrm{n}$ & $2,37 \pm 1,50$ & $2,30 \pm 0,88$ & $0,498^{\#}$ \\
\hline Hipnóticos/Antidepressivos, \% & $6(20)$ & $7(23,3)$ & $0,754^{\&}$ \\
\hline Diuréticos, \% & $14(46,7)$ & $16(53,3)$ & $0,606^{\&}$ \\
\hline Coluna lombar, T-score & $-2,83 \pm 1,07$ & $-2,62 \pm 1,12$ & $0,470^{\#}$ \\
\hline Colo de fêmur, T-score & $-2,70 \pm 0,75$ & $-2,75 \pm 0,90$ & $0,821^{\#}$ \\
\hline Fêmur Total, T-score & $-2,10 \pm 1,26$ & $-2,10 \pm 1,09$ & $0,990^{\#}$ \\
\hline \multicolumn{4}{|l|}{ * Teste t-student } \\
\hline \multicolumn{4}{|l|}{ \# Teste Mann-Whitney } \\
\hline \multicolumn{4}{|l|}{ \& Teste qui-quadrado } \\
\hline Dados expressos em médias $\pm D$ & porcentagem & & \\
\hline
\end{tabular}


TABELA II. Dados no início do estudo do escore da Escala de Berg (BBS), número de pacientes que não completaram o Teste Clínico para Avaliação da Integração Sensorial no Equilíbrio (CTSIB: condição 1- olhos abertos e superfície estável; condição 2- olhos vendados e superfície estável; condição 3- olhos abertos, superfície estável e cúpula visual; condição 4- olhos abertos e superfície instável; condição 5- olhos vendados e superfície instável; condição 6- olhos abertos, superfície instável e cúpula visual), Teste do Tempo de Ir e Vir (TUGT) e número de quedas/paciente no último ano, nos grupos Intervenção e Controle.

\begin{tabular}{lccc}
\hline Variáveis & $\begin{array}{c}\text { Intervenção } \\
(\mathrm{n}=30)\end{array}$ & $\begin{array}{c}\text { Controle } \\
(\mathrm{n}=30)\end{array}$ & $\mathrm{P}$ \\
\hline BBS, escore & $48,80 \pm 4,10$ & $48,13 \pm 5,36$ & $0,900^{\#}$ \\
CTSIB condição 1, n (\%) & $0(0)$ & $1(3,3)$ & $1,000^{\&}$ \\
CTSIB condição 2, n (\%) & $2(6,7)$ & $1(3,3)$ & $1,000^{\&}$ \\
CTSIB condição 3, n (\%) & $2(6,7)$ & $2(6,7)$ & $1,000^{\&}$ \\
CTSIB condição 4, n (\%) & $4(13,3)$ & $4(13,3)$ & $1,000^{\&}$ \\
CTSIB condição 5, n (\%) & $15(50)$ & $12(40)$ & $0,604^{\&}$ \\
CTSIB condição 6, n (\%) & $12(40)$ & $9(30)$ & $0,589^{\&}$ \\
TUGT, segundos & $14,31 \pm 4,03$ & $13,86 \pm 3,43$ & $0,610^{\#}$ \\
Quedas/paciente último ano, n & $1,20 \pm 1,88$ & $0,87 \pm 0,86$ & $0,745^{\#}$ \\
\hline \# Teste Mann-Witney & & & \\
\& Teste exato de Fisher & & & \\
Dados expressos em médias \pm DP ou porcentagem. & & \\
& & & \\
\hline
\end{tabular}




\section{Taxa de adesão e comparecimento}

Uma alta taxa de comparecimento foi observada. Sessenta por cento das pacientes participaram de todas as sessões de exercícios no clube. As faltas ocorreram com justificativas de: consulta médica, realização de exames ou razões pessoais. Com relação ao exercício em casa: 76,67\% dos pacientes realizaram pelo menos uma vez por semana, $40 \%$, todos os dias e $36,67 \%$ de uma a quatro vezes por semana.

\section{Comparação entre os Grupos Intervenção e Controle}

Como esperado, uma diferença significante no escore da Escala de Berg (avaliação final-inicial) foi maior no grupo que sofreu intervenção $(5,5 \pm 5,67$ vs. $-0,5 \pm 4,88, \mathrm{P}<0,001)$ (Tabela III).

De maneira similar, a porcentagem de pacientes, no grupo Intervenção, que melhorou o equilíbrio estático em duas condições sensoriais (CTSIB: condição 5- olhos vendados e superfície instável; condição 6- olhos abertos, superfície instável e cúpula visual) foi estatisticamente significante quando comparado com o Controle (CTSIB condição 5: 13 vs. 1 paciente, $\mathrm{P}<0.001$; CTSIB condição 6: 12pacientes vs. 1 paciente, $\mathrm{P}<0.001$ ) (Tabela III).

Igualmente, a melhora da mobilidade funcional, mensurada pelo TUGT (avaliação final - inicial), foi observada no grupo Intervenção comparada com o Controle $(-3,65 \pm 3,61$ vs. $+2,27 \pm 7,18, \mathrm{P}<0.001)$ (Tabela III).

Paralelamente a essa melhora no estado funcional, houve uma redução de quedas/paciente (avaliação final - inicial) no grupo Intervenção 
comparado com o Controle $(-0,77 \pm 1,76$ vs. $+0,03 \pm 0,98, \mathrm{P}=0.018)$ (Tabela III).

TABELA III. Diferenças (avaliação final - avaliação inicial) no escore da Escala de Berg (BBS), número de pacientes que apresentaram melhora no Teste Clínico para Avaliação da Integração Sensorial no Equilíbrio (CTSIB condição 5: olhos vendados e superfície instável e condição 6: olhos abertos, superfície instável e cúpula visual); Teste do Tempo de Ir e Vir (TUGT) e redução das quedas/paciente nos grupos Intervenção e Controle.

\begin{tabular}{lccc}
\hline Variáveis & $\begin{array}{c}\text { Intervenção } \\
(\mathrm{n}=30)\end{array}$ & $\begin{array}{c}\text { Controle } \\
(\mathrm{n}=30)\end{array}$ & $\mathrm{P}$ \\
\hline Diferença BBS, escore & $5,5 \pm 5,67$ & $-0,5 \pm 4,88$ & $<0,001^{\#}$ \\
CTSIB condição 5, n (\%) & $13(43,3)$ & $1(3,3)$ & $<0,001^{\&}$ \\
CTSIB condição 6, n (\%) & $12(40)$ & $1(3,3)$ & $0,001^{\&}$ \\
Diferença TUGT, segundos & $-3,65 \pm 3,61$ & $+2,27 \pm 7,18$ & $<0,001^{\#}$ \\
Diferença de Quedas/paciente, $\mathrm{n}$ & $0,77 \pm 1,76$ & $0,03 \pm 0,96$ & $0,018^{\#}$
\end{tabular}

\# Teste Mann-Whitney test

\& Teste Chi-square

Dados expressos em médias \pm DP ou porcentagem. 
DISCUSSÃO 
Poucos estudos têm sido desenvolvidos sobre o treino de equilíbrio em pacientes com osteoporose. Nosso estudo longitudinal prospectivo demonstrou que um programa de treino de equilíbrio realizado durante 12 meses foi efetivo para melhorar o equilíbrio funcional e estático, a mobilidade e redução do número de quedas em mulheres idosas com osteoporose.

No presente estudo, a melhora em relação ao equilíbrio funcional foi demonstrada pelo aumento do escore da Escala de Berg (BBS) no final da avaliação do grupo submetido ao programa de treino de equilíbrio (Grupo Intervenção). Resultados semelhantes foram demonstrados no estudo de Melzer e cols. ${ }^{32}$, em que os pacientes que participaram do treino de equilíbrio obtiveram 64\% de melhora (em 3 meses). Esses autores demonstraram que o grupo que fez o treino de equilíbrio teve um melhor desempenho do que o grupo submetido ao treino de força muscular.

Particularmente, os programas que enfatizam o treino do equilíbrio são mais efetivos na melhora do paciente do que aqueles que consistem principalmente de exercícios aeróbicos, de força muscular ou flexibilidade ${ }^{33}$.

Embora seja complexo avaliar a efetividade dos diferentes tipos de exercícios $^{34}$, o treino de equilíbrio tem mostrado resultados benéficos com redução no risco de quedas ${ }^{35}$.

Outro resultado positivo em nosso estudo foi a melhora de duas condições do Teste Clínico para Avaliação da Integração Sensorial no Equilíbrio (CTSIB condição 5: olhos abertos e superfície instável e condição 6: olhos abertos, superfície instável e cúpula visual) em quase metade dos 
pacientes. Carter e cols. ${ }^{24}$, utilizando o treino de força muscular, em vez do treino de equilíbrio, obtiveram uma melhora do equilíbrio estático, todavia, em somente $6,3 \%$ dos pacientes. Assim, esses achados sugerem que o treino de equilíbrio leva a resultados positivos mais evidentes no equilíbrio estático quando comparado com o treino de força muscular.

Em relação à mobilidade funcional, houve uma melhora demonstrada pela redução no tempo do teste TUGT em pacientes submetidos à intervenção. Esses resultados são relevantes, uma vez que pesquisas demonstram que o comprometimento da mobilidade aumenta o risco (três a cinco vezes) de dependência nas atividades de vida diária (AVDs) ${ }^{36}$. Isso não é surpreendente considerando que a mobilidade é um componente importante das AVDs, como por exemplo: ir ao shopping, a supermercados, ao médico ou ao cinema. O aumento da dependência pode levar à institucionalização e à diminuição da qualidade de vida. Um bom equilíbrio é considerado fundamental para melhorar a mobilidade e prevenir quedas ${ }^{36}$.

Paralelamente a melhora do estado funcional, observou-se uma importante redução na freqüência das quedas. Embora o efeito do exercício na prevenção de quedas no idoso ainda não tenha sido comprovado, estudos mostram consistentemente que a atividade física reduz o seu risco em $40 \%{ }^{37}$. Em um consenso sobre prevenção de quedas no idoso, somente o treino de equilíbrio foi recomendado para prevenir quedas ${ }^{38}$.

Muitos estudos que mostram a melhora no equilíbrio e na mobilidade têm características semelhantes em seu exercício. Isso sugere que o 
conteúdo e a intensidade do programa de exercício pode ser mais importante do que outras variáveis da intervenção ${ }^{39}$. Entretanto, diferenças na administração do programa de exercício, a experiência do profissional que aplica o exercício, o local em que são feitas as sessões, se o mesmo é aplicado em casa ou no hospital, a duração do programa de exercício, se o exercício é conduzido em grupo ou realizado individualmente são parâmetros fundamentais que influenciam no sucesso do programa de exercício ${ }^{39}$.

Outro fator importante que contribuiu para o sucesso do programa de exercício foi a aderência, o que contraria o estudo de Forcan ${ }^{40}$ que mostra que a aderência ao exercício em idosos é fraca. No presente estudo, observou-se uma boa aderência sendo que mais da metade das pacientes estava presente em todas as sessões. Esse achado é semelhante a outros estudos que mostram aderência de $97,5 \%{ }^{17}$.

O sucesso da aderência foi secundário a diversos fatores fundamentais, dentre eles, é possível citar:

O local onde foram feitos os exercícios, por ser um ambiente seguro, agradável e natural;

A supervisão de um fisioterapeuta nas sessões, a maneira como ele cobrava os exercícios a serem feitos em casa, o entusiasmo e a dinâmica com que conduzia o treino resultou na fidelidade da realização dos exercícios pelas pacientes;

O exercício feito em grupo, a oportunidade para interação social reduz o sentimento de isolamento. O suporte social tem sido considerado importante 
nas atividades em grupo ${ }^{50}$. Isso também ajuda a sustentar a aderência e a efetividade das sessões dos exercícios feitos em casa semanalmente ${ }^{35}$. Semelhantemente ao nosso estudo, Robitaille e cols. mostraram que o programa de exercícios, feito em grupo, melhora o equilíbrio em idosos da comunidade $^{41}$. Houve uma identificação entre as pacientes por terem a mesma idade e doenças semelhantes;

Além disso, uma vez por mês, após o treino, eram realizadas confraternizações que propiciavam maior interação entre as pacientes, criando-se um vínculo entre as pacientes, notou-se uma preocupação de cobrança entre elas de fazerem os exercícios. Collelo e cols. demonstraram que a orientação e a prática de exercício feita em grupo têm efeitos positivos, tornando o idoso mais independente e seguro nas atividades de vida diária ${ }^{55}$;

Outro fator considerável foi a utilização de um manual com instruções e ilustrações para a realização dos exercícios em casa. Essa iniciativa contribuiu para a continuidade e aderência dos exercícios feitos. Cada exercício foi prescrito e ilustrado apropriadamente por um fisioterapeuta, dando ao paciente um suporte adequado. Notou-se que as pacientes se sentiram valorizadas com a orientação didática. Panfletos descritivos e ilustrativos têm sido utilizados em alguns trabalhos complementados por programas de exercício em casa com resultados positivos ${ }^{3,42}$.

Os resultados positivos também podem estar relacionados ao estado de saúde não muito comprometido das pacientes. Buchner e cols. ${ }^{43}$ mostraram 
que o exercício pode ter efeitos benéficos na saúde e no risco de quedas em alguns subgrupos de idosos.

Os benefícios físicos e psicológicos da regularidade das sessões e o meio ambiente nunca devem ser ignorados. Estimular as estratégias e ter habilidade nas transferências, mantendo o entusiasmo durante o exercício, são fatores que devem e podem ser praticados seguramente em grupo ${ }^{35}$.

Embora este estudo não tenha utilizado a posturografia dinâmica computadorizada $^{44}$ para subsidiar os resultados obtidos, mostrou-se pelas escalas e testes confiáveis e reproduzíveis ${ }^{26,28,29}$ que o treino de equilíbrio foi eficaz.

Concomitantemente, observou-se uma boa aderência aos exercícios realizados em casa, sendo que quase metade das pacientes os executava todos os dias. 0 programa de exercícios feito em casa também melhora o desempenho físico, o equilíbrio e reduz as quedas ${ }^{38,52,53}$. 
CONCLUSÃO 
O treino de equilíbrio realizado por 12 meses em mulheres idosas com osteoporose foi muito efetivo na melhora:

- do equilíbrio funcional,

- do equilíbrio estático,

- da mobilidade funcional e

- na redução de quedas. 
REFERÊNCIAS 
1. Kuczynski, M, Ostrowska, B: Understanding falls in osteoporosis: the viscoelastic modeling perspective. Gait \& Posture 23: 51-8, 2006.

2. Sinaki M, Brey RH, Hughes CA, Larson DR, Kaufman KR: Balance disorder and increased risk of falls in osteoporosis and kyphosis: significance of kyphotic posture and muscle strength. Osteoporos Int 16: 1004-10, 2005.

3. Suzuki $\mathrm{T}$, Kim $\mathrm{H}$, Yoshida $\mathrm{H}$, Ishizaki $\mathrm{T}$ : Randomized controlled trial of exercise intervention for the prevention of falls in community-dwelling elderly Japanese women. J Bone Miner Metab 22: 602-11, 2004.

4. Barraff LJ, Della PR, Willians N. et al.: Practice guideline for the ED management of falls in community-dwelling elderly persons. Ann Emerg Med 30: 480-92, 1997.

5. Ashley MJ, Gryfe CI, Amies A: A longitudinal study of falls in an elderly population, II: some circumstances of falling. Age Ageing 6: 211-20, 1977.

6. Silsupadol P, Siu KC, Shumway-Cook A, Woollacott MH: Training balance under single and dual-task conditions in older adults with balance impairment. Phys Ther 86: 269-81, 2006.

7. Campbell AJ, Robertson MC, Gardner MM, Norton RN, Buchner DM: Psychotropic medicine withdrawal and a home-based exercise program to prevent falls: a randomized controlled trial. J Am Geriatr Soc 47: 8503, 1999. 
8. Overstall PW, Exton-Smith AN, Imms FJ, Johnson AL: Falls in the elderly related postural imbalance. Br Med J 1: 261-64, 1977.

9. Nelson RC, Amin MA: Falls in elderly. Emerg Med Clin North Am 8: 30924, 1990.

10. Campbell AJ, Borrie MJ, Spears GF, et al.: Circumstances and consequences of falls experienced by a community population 70 years and over during a prospective study. Age ageing 19: 136-41, 1990.

11. Tinetti ME, Baker, DI, McAvay G et al.: A multifactorial intervention to reduce the risk of falling among elderly people living in the community. N Engl J Med 331: 821-27, 1994.

12. Duncan PW, Studenski S, Chandler J, Prescott B: Functional reach: predictive validity in a sample of elderly male veterans. J Gerontol 47: 93-8, 1992.

13. Hindmarsh JJ, Estes EH Jr: Falls in older persons. Causes and interventions. Arch Intern Med 149: 2217-22, 1989.

14. Horak FB, Shupert CL, Mirka A: A components of dyscontrol in the elderly: A review. Neurobiol Aging 10: 727-38, 1989.

15. Close JCT, Glucksman E: Falls in elderly: What can be dobe? Med J Aust 173: 176-7, 2000.

16. Steadman J, Donaldson N, Kalra L: A randomized controlled trial of an enhanced balance training program to improve mobility and reduce falls in elderly patients. JAGS 51: 847-52, 2003. 
17. Sihvonen S, Sipilä S, Taskinen S, Era P: Fall incidence in frail olderwomen after individualized visual feedback-based balance training. Gerontology 50: 411-6, 2004.

18. Nowalk MP, Prendergast JM, Bayles CM, D'amico FJ, Colvin GC: A randomized trial of exercises programs among older individuals living in two long-term care facilities: The Falls-FREE Program. J Am Geriatr Soc 49: 859-65, 2001.

19. Judge J, Whipple RH, Wolfson LI et al. Effects of balance exercises on isokinetic strength in older persons. J Am Geriatr Soc 42:937-46, 1994.

20. Nitz JC, Choy NL: The efficacy of a specific balance-strategy training programme for preventing falls among older people: a pilot randomised controlled trial. Age and Ageing 33: 52-58, 2004.

21. Seidler RD, Martin PE: The effects of short term balance training on the postural control of older adults. Gait Posture 6: 224-36, 1997.

22. Carter ND, Khan KM, Petit MA et al.: Results of a 10 week community based strength and balance training programme to reduce fall risk factors: a randomized controlled trial in 65-75 year old women with osteoporosis. Br J Sports Med 35: 348-51, 2001.

23. WHO World Health Organization: Assessment of osteoporotic fracture risk and its role in screening for menopausal osteoporosis, Geneva, WHO Technical Report Series, 1994. 
24. Carter ND, Khan, KM, McKay HA, Petit MA, Waterman C, Heinonen A, et al. Community-based exercise program reduces risk factors for falls in 65- to 75-year-old women with osteoporosis: randomized controlled trial. JAMC 167: 997-1003, 2002.

25. American College of Sports Medicine: Guidelines for Exercise Testing and Prescription, $5^{\text {th }}$ Ed. Baltimore: Williams and Wilkins, pp. 1-373, 1995.

26. Miyamoto ST, Lombardi Junior I, Berg KO, Ramos LR, Natour J: Brazilian version of the Berg balance scale. Braz J Med Biol Res 37: 1411-1421, 2004.

27. Whitney SL, Poole JL, Cass SP: A review of balance instruments for older adults. Am J Occup Ther 52: 666-71, 1998.

28. Shumway-Cook A, Horak FB: Assessing the influence of sensory interaction on balance, suggestion from the field. Phys Ther 66: 1548$50,1986$.

29. Podsiadlo D \& Richardson S: The Timed "Up \& Go": a test of basic functional mobility for frail elderly persons. JAGS 39: 142-8, 1991.

30. Tinetti ME, Speechley M, Ginter SF: Risk factors for falls among elderly persons living in the community. N Engl J Med 319: 1701-7 1988.

31. Van Norman KA: Human Kinetics. Champaign, IL, USA, pp 41-66, 1995.

32. Melzer I, Benjuya N, Kaplanski J: Effect of physical training on postural control of elderly. Harefuah 114: 839-44, 2005. 
33. Rogers $\mathrm{ME}$, Fernandez JE, Bohlken RM: Training to reduce postural sway and increase functional reach in the elderly. J Occup Rehabil 11: 291-8, 2001.

34. Chang JT, Morton SC, Rubenstein LZ, Mojica WA, Maglione M, Suttorp MJ, Roth EA, Shekelle PG: Interventions for the prevention of falls in older adults: systematic review and meta-analysis of randomized clinical trials. BMJ 328: 680-3, 2004.

35. Skelton DA.: Effects of physical activity on postural stability. Age Ageing 30: 33-9, 2001.

36. Frank JS, Patla A E: Balance and mobility challenges in older adults: Implications for preserving community mobility. Am J Prev Med 25:15763, 2003.

37. Barnett A, Smith B, Lord SR, Williams M, Baumand A: Community-based group exercise improves balance and reduces falls in at-risk older people: a randomized controlled trial. Age ageing 32:407-14, 2003.

38. Feder G, Cryer C, Donovan S: Guidelines for the prevention of falls in older people. London: Queen Mary and Westfield College, 1998.

39. Means KM, Rodell DE, O'Sullivan PS: Balance, mobility, and falls among community-dwelling elderly persons: effects of a rehabilitation exercise program. Am J Phys Med Rehabil 84: 238-50, 2005. 
40. Forcan R, Pumper B, Smyth N, Wirkkala H, Ciol MA, Shumway-Cook A: Exercise adherence following physical therapy intervention in older adults with impaired balance. Phys Ther 86: 401-10, 2006.

41. Robitaille $\mathrm{Y}$, Laforest $S$, Fournier $M$, et al.: Moving forward in fall prevention: an intervention to improve balance among older adults in real-world settings. Am J Public Health 95: 2049-56, 2005.

42. King $M B$, Whipple $\mathrm{RH}$, Gruman $\mathrm{CA}$, Judge JO: He performance enhancement project: improving physical performance in older persons. Arch Phys Med Rehabil 83: 1060-1069, 2002.

43. Buchner DM, Cress ME, de Lateur BJ, Esselman PC, Margherita AJ, Price R, Wagner EH: The effect of strength and endurance training of gait, balance, fall risk, and health services use in community-living older adults. J Gerontol A Biol Sci Med Sci 52: 218-24, 1997.

44. Sinaki M, Brey RH, Hughes CA, Larson DR, Kaufman KR (2005) Significant reduction in risk of falls and back pain osteoporotic-kyphotic women through a spinal proprioceptive extension exercise dynamic (SPEED) program. Mayo Clinic Proceedings 80:849-55. 
ANEXO 1

\section{APROVAÇÃO DA COMISSÃO DE ÉTICA}

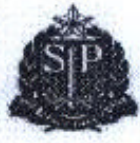

HOSPITAL DAS CLINICAS

DA FACULDADE DE MEOICINA DA UNIVERSIOADE OE SAO PAULO CALKA POSTAL, 8091 - SAO PAULO - BRASHL

\section{DIRETORIA CLÍNICA}

\section{Comissão de Ética para Análise de Projetos de Pesquisa}

\section{APROVACÃO}

A Comissão de Ética para Análise de Projetos de Pesquisa - CAPPesq da Diretoria Clínica do Hospital das Clínicas e da Faculdade de Medicina da Universidade de São Paulo, em sessão de 29.05.03, APROVOU o Protocolo de Pesquisa $n^{\circ} 218 / 03$, intitulado: "Influência de um programa de orientação à atividade física na qualidade de vida e no equilibrio funcional de pacientes com osteoporose senil" apresentado pelo Departamento de CLINICA MÉDICA, bem como o Termo de Consentimento Livre e Esclarecido.

Pesquisador(a) Responsável: PROFA. ORA. ROSA MARIA RODRIGUES PEREIRA

Pesquisador(a) Executante: SRA. MELISA MOREIRA MADUREIRA

CAPPesq, 29 de Maio de 2003.

$$
\text { L14. } 6 \text { Cll }_{0}
$$

PROF. DR. EUCLIDES AYRES DE CASTILHO Presidente da Comissāo de Ética para Análise de Projetos de Pesquisa

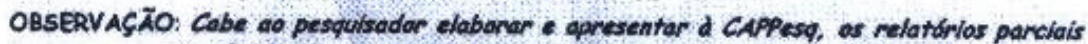
- final sobre a pesquisa (Resolugido da Conselho Nacional de Saúde $n^{\circ} 196$, de 10,10.1996. inciso IX,2, letra "c? 
ANEXO 2

HOSPITAL DAS CLÍNICAS

DA

FACULDADE DE MEDICINA DA UNIVERSIDADE DE SÃO PAULO

\section{TERMO DE CONSENTIMENTO LIVRE E ESCLARECIDO}

(Instruções para preenchimento no verso)

\section{I - DADOS DE IDENTIFICAÇÃO DO SUJEITO DA PESQUISA OU RESPONSÁVEL LEGAL}

1. NOME DO PACIENTE:

DOCUMENTO DE IDENTIDADE NO :

DATA NASCIMENTO:

SEXO : $M ? \quad F ?$

ENDEREÇO

BAIRRO: CIDADE:

CEP: $\quad$ TELEFONE: DDD ( )

2. RESPONSÁVEL LEGAL:

NATUREZA (grau de parentesco, tutor, curador etc.)

DOCUMENTO DE IDENTIDADE :

DATA NASCIMENTO: ....................

ENDEREÇO:

BAIRRO:

CIDADE:

SEXO: $M$ ? $F$ ?

CEP:

TELEFONE: DDD ( )

\section{II - DADOS SOBRE A PESQUISA CIENTÍFICA}

TÍTULO DO PROTOCOLO DE PESQUISA:

Influência de um Programa de Orientação à Atividade Física na Qualidade de Vida e no Equilíbrio Funcional de Pacientes com Osteoporose Senil.

PESQUISADOR: Dra. Rosa Maria Rodrigues Pereira

CARGO/FUNÇÃO: Profa. Assistente INSCRIÇÃO CONSELHO REGIONAL No 45920

UNIDADE DO HCFMUSP: LIM-17

3. AVALIAÇÃO DO RISCO DA PESQUISA:

$\begin{array}{lll}\text { SEM RISCO ? } & \text { RISCO MÍNIMO } \\ \text { RISCO BAIXO } \mathbf{X} & \text { RISCO MAIOR } & ?\end{array}$

(Leve cansaço, sem causar dor. O risco de uma eventual queda durante o exame é mínimo, uma vez que sua segurança será garantida pelo fisioterapeuta e pelo ambiente).

4. DURAÇÃO DA PESQUISA : 2 anos. 


\section{III - REGISTRO DAS EXPLICAÇÕES DO PESQUISADOR AO PACIENTE OU SEU REPRESENTANTE LEGAL SOBRE A PESQUISA, CONSIGNANDO:}

\section{GRUPO INTERVENÇÃO}

1. Justificativa e os objetivos da pesquisa; 2. procedimentos que serão utilizados e propósitos, incluindo a identificação dos procedimentos que são experimentais; 3 . desconfortos e riscos esperados; 4. benefícios que poderão ser obtidos; 5 . procedimentos alternativos que possam ser vantajosos para o indivíduo.

Para o paciente que irá fazer exercício:

1. A qualidade de vida, o equilíbrio corporal e a postura são muito importantes para a nossa saúde. No entanto, esses fatores podem ser alterados pelo envelhecimento e por uma doença comum durante este período, denominada osteoporose (osso fraco). Indivíduos, acima de 65 anos, que têm osteoporose podem piorar ainda mais a qualidade de vida, a postura e o equilíbrio corporal, uma vez que estão mais sujeitos a quedas e fraturas. O exercício parece melhorar a vida do indivíduo acima de 65 anos no que diz respeito a qualidade de vida, a postura, ao equilíbrio corporal e à força muscular. Dessa forma, este trabalho irá verificar a qualidade de vida e o equilíbrio corporal de mulheres na terceira idade, com osteoporose antes, e após um ano de atividade física.

2. Para isso, gostaríamos de convidá-la a participar deste estudo que consiste de uma avaliação postural, dois questionários e três testes de equilíbrio. Toda a avaliação será realizada por um fisioterapeuta no período de aproximadamente 1 hora, após a avaliação clínica habitual, que será realizada pelo seu médico. Os questionários serão compostos por perguntas sobre dificuldades nas tarefas do dia-a-dia, queixas gerais e qualidade de vida. Haverá uma avaliação postural fotográfica e alguns testes de equilíbrio corporal que imitam nossas atividades diárias (levantar e sentar na cadeira, ficar em pé por dois minutos, permanecer em pé com olhos fechados, andar em um trajeto de três metros, entre outros). O programa de exercícios seguirá a seqüência de: alongamentos; aquecimento (caminhada), condicionamento físico; exercícios de fortalecimento muscular; exercícios específicos para melhorar o equilíbrio (ficar sobre uma perna, colocar um pe na frente do outro, andar levantando a perna); e, por fim.

3. Não são esperados desconfortos ou riscos, exceto a possibilidade de tontura e desequilíbrio momentâneos, um leve cansaço, sem causar dor. O risco de uma eventual queda durante o exame é mínimo, uma vez que sua segurança será garantida pelo fisioterapeuta e pelo ambiente.

4. Essa avaliação pela qual a senhora se submeterá, proporcionará a sua inclusão, gratuitamente, em uma abordagem de reabilitação por uma equipe especializada do Serviço de Reabilitação do Hospital das Clínicas da Faculdade de Medicina da Universidade de São Paulo, serviço este que visa orientar exercícios para melhorar a qualidade de vida, a postura e o equilíbrio corporal.

5. Ao participar do programa de orientação à atividade física, o paciente poderá, portanto, melhorar sua qualidade de vida, sua postura e seu equilíbrio corporal. 


\section{GRUPO CONTROLE}

\section{III -REGISTRO DAS EXPLICAÇÕES DO PESQUISADOR AO PACIENTE OU SEU REPRESENTANTE LEGAL SOBRE A PESQUISA, CONSIGNANDO:}

1. Justificativa e os objetivos da pesquisa; 2. procedimentos que serão utilizados e propósitos, incluindo a identificação dos procedimentos que são experimentais; 3. desconfortos e riscos esperados; 4 . benefícios que poderão ser obtidos; 5 . procedimentos alternativos que possam ser vantajosos para o indivíduo.

Para o paciente, sem atividade física:

1. A qualidade de vida, o equilíbrio corporal e a postura são muito importantes para a nossa saúde. No entanto, esses fatores podem ser alterados pelo envelhecimento e por uma doença comum durante este período, denominada osteoporose (osso fraco). Indivíduos, acima de 65 anos, que têm osteoporose podem piorar ainda mais a qualidade de vida, a postura e o equilíbrio corporal, uma vez que estão mais sujeitos a quedas e fraturas. O exercício parece melhorar a vida do indivíduo acima de 65 anos no que diz respeito a qualidade de vida, a postura, ao equilíbrio corporal e a força muscular. Dessa forma, este trabalho irá verificar a qualidade de vida e o equilíbrio corporal de mulheres na terceira idade com osteoporose antes e após um ano.

2. Para isso, gostaríamos de convidá-la a participar deste estudo que consiste de uma avaliação postural, dois questionários e três testes de equilíbrio. Toda a avaliação será realizada por um fisioterapeuta no período de aproximadamente 1 hora, após a avaliação clínica habitual, que será realizada pelo seu médico. Os questionários serão compostos por perguntas sobre dificuldades nas tarefas do dia-a-dia, queixas gerais e qualidade de vida. Haverá uma avaliação postural fotográfica e alguns testes de equilíbrio corporal que imitam nossas atividades diárias (levantar e sentar na cadeira, ficar de pé por dois minutos, permanecer de pé com olhos fechados, andar em um trajeto de três metros, entre outros).

3. Não são esperados desconfortos ou riscos, exceto a possibilidade de tontura e desequilíbrio momentâneos, um leve cansaço, sem causar dor. O risco de uma eventual queda durante o exame é mínimo, uma vez que sua segurança será garantida pelo fisioterapeuta e pelo ambiente.

4 e 5. Não há benefícios e vantagens para o paciente que participar desta pesquisa. 


\section{IV - ESCLARECIMENTOS DADOS PELO PESQUISADOR SOBRE GARANTIAS DO SUJEITO DA PESQUISA:}

1. Acesso, a qualquer tempo, às informações sobre procedimentos, riscos e benefícios relacionados à pesquisa, inclusive para dirimir eventuais dúvidas; 2 . liberdade de retirar seu consentimento a qualquer momento e de deixar de participar do estudo, sem que isto traga prejuízo à continuidade da assistência; 3. salvaguarda da confidencialidade, sigilo e privacidade; 4. disponibilidade de assistência no HCFMUSP, por eventuais danos à saúde, decorrentes da pesquisa; 5. viabilidade de indenização por eventuais danos à saúde decorrentes da pesquisa.

2. O paciente ou responsável terá acesso para questões sobre seus direitos como indivíduo do estudo, entrando em contato com a Comissão de Ética do Hospital das Clínicas, no telefone 3069-6442, para qualquer pergunta sobre o estudo, entrar em contato com a médica do estudo, Dra. Rosa Maria Rodrigues Pereira, no telefone 3066-7213 ou a fisioterapeuta Melisa Moreira Madureira, no telefone 3069-7227.

3. A participação do paciente neste estudo é voluntária, podendo escolher não fazer parte deste estudo.

4. Caso o paciente concorde em participar deste estudo, isto será mantido em sigilo. Esta pesquisa não envolve dano à saúde do paciente. O nome da senhora será mantido em sigilo, e a senhora poderá retirar seu consentimento no momento em que achar necessário. Serão esclarecidas todas as suas dúvidas, inclusive durante o exame. Os dados obtidos serão utilizados apenas para finalidade de pesquisa, sendo garantido total sigilo de minha identidade.

5. Fui informado que poderei desistir de participar do projeto em qualquer momento, sendo que minha desistência não terá qualquer implicação na continuidade de tratamento que eu esteja recebendo no Hospital das Clínicas da FMUSP.

6. Esta pesquisa não envolve danos à saúde do paciente. 
V - INFORMAÇÕES DE NOMES, ENDEREÇOS E TELEFONES DOS RESPONSÁVEIS PELO ACOMPANHAMENTO DA PESQUISA, PARA CONTATO EM CASO DE INTERCORRÊNCIAS CLÍNICAS E REAÇÕES ADVERSAS.

Dra. Rosa Maria Rodrigues Pereira - Faculdade de Medicina da USP

$3^{\circ}$. andar - Reumatologia - Sala 3107

Fone: (011) 3066-7213 / 3721-0178.

\section{VI - OBSERVAÇÕES COMPLEMENTARES:}

\section{VII - CONSENTIMENTO PÓS-ESCLARECIDO}

Declaro que, após convenientemente esclarecido pelo pesquisador e ter entendido o que me foi explicado, consinto em participar do presente Protocolo de Pesquisa.

São Paulo, de de 2003. 


\section{FICHA DE COLETA DE DADOS}

ANEXO 3

Início da avaliação -

Nome:

$\mathrm{RG} / \mathrm{HC}$

Idade:

Peso:

Altura:

IMC:

Mora só? Profissão:

Data da menopausa:

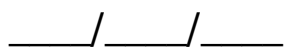

Percepção subjetiva da visão e audição - Acuidade visual diminuída (S) (N)

Diminuição da audição

$(\mathrm{S})(\mathrm{N})$

Possui alguma outra doença?

( ) DM

( ) HAS

( ) Alteração vestibular Sente tontura? (S) (N)

( ) Outras doenças

Fumante: (S) (N)（）Pregressa（）Atual - Número de cigarros/dia ( ) Álcool: (S) (N) Com que freqüência?

Nutrição: Copos de leite dia Copos de yorgute /dia Fatias queijo /dia /dia 


\begin{tabular}{|l|l|}
\hline \multicolumn{1}{|c|}{ Medicações } & Tempo \\
\hline TRH & \\
\hline Bisfosfonato & \\
\hline Cálcio & \\
\hline Vitam. D & \\
\hline Medicações: (hipotensores, hipnóticos e antidepressivos) & \\
\hline
\end{tabular}

Atividade Física: (S) (N) Tipos:

Freqüência: (1x)sem (2x)sem (3x)sem (4x)sem (5x)sem ( ) Todos os dias

Caiu nos últimos 12 meses? (S) (N)

Quantas vezes? (0) Nenhuma (1) (2) (3) (4)

Antecedente de fraturas nos últimos 5 anos: (S) (N)

Fraturas prévias: $(\mathrm{S})(\mathrm{N})$ Tipos:

Diagnóstico densitométrico /2002 Peso Altura

\begin{tabular}{|l|l|l|}
\hline L1-L4= & T= & Z= \\
\hline Colo fêmur= & T= & $Z=$ \\
\hline Fêmur Total= & T= & $Z=$ \\
\hline
\end{tabular}


Diagnóstico densitométrico /2003 Peso Altura

\begin{tabular}{|l|l|l|}
\hline L1-L4= & T= & $Z=$ \\
\hline Colo fêmur= & T= & $Z=$ \\
\hline Fêmur Total= & T= & $Z=$ \\
\hline
\end{tabular}

Calçado habitual: ( ) sapato baixo ( ) sandália ( ) de salto ( ) tênis 


\section{AVALIAÇÃO DO EQUILÍBRIO FUNCIONAL}

Escala de Equilíbrio de Berg (BBS) (MIYAMOTO et al, 2003)

\section{INSTRUÇõES GERAIS}

Por favor, demonstrar cada tarefa e/ou dar as instruções como estão descritas. Ao pontuar, registrar a categoria de resposta mais baixa, que se aplica a cada item.

Na maioria dos itens, pede-se ao paciente para manter uma determinada posição durante um tempo específico. Progressivamente mais pontos são deduzidos, se o tempo ou à distância não forem atingidos, se o paciente precisar de supervisão (o examinador necessita ficar bem próximo do paciente) ou fizer uso de apoio externo ou receber ajuda do examinador. Os pacientes devem entender que eles precisam manter o equilíbrio enquanto realizam as tarefas. As escolhas sobre qual perna ficar em pé ou qual distância alcançar, ficará a critério do paciente. Um julgamento pobre irá influenciar adversamente o desempenho e o escore do paciente.

Os equipamentos necessários para realizar os testes são: um cronômetro ou um relógio com ponteiro de segundos, uma régua ou outro indicador de: $5 ; 12,5$ e $25 \mathrm{~cm}$. As cadeiras utilizadas para o teste devem ter uma altura adequada. Um banquinho ou uma escada (com degraus de altura padrão) pode ser usado para o item 12.

\section{POSIÇÃo SENTADA PARA POSIÇÃO EM PÉ}

INSTRUÇÕES: Por favor, levante-se. Tente não usar suas mãos para se apoiar.

( ) 4 capaz de levantar-se sem utilizar as mãos e estabilizar-se independentemente

( ) 3 capaz de levantar-se independentemente utilizando as mãos

( ) 2 capaz de levantar-se utilizando as mãos após diversas tentativas

( ) 1 necessita de ajuda mínima para levantar-se ou estabilizar-se

( ) 0 necessita de ajuda moderada ou máxima para levantar-se

\section{PERMANECER EM PÉ SEM APOIO}

INSTRUÇÕES: Por favor, fique em pé por 2 minutos sem se apoiar.

( ) 4 capaz de permanecer em pé com segurança por 2 minutos

( ) 3 capaz de permanecer em pé por 2 minutos com supervisão

( ) 2 capaz de permanecer em pé por 30 segundos sem apoio

( ) 1 necessita de várias tentativas para permanecer em pé por 30 segundos sem apoio

( ) 0 incapaz de permanecer em pé por 30 segundos sem apoio

Se o paciente for capaz de permanecer em pé por 2 minutos sem apoio, dê o número total de pontos o item $\mathrm{N}^{\circ} 3$. Continue com o item $\mathrm{N}^{\circ} 4$. 


\section{PERmanecer SENTAdo Sem apoio nas Costas, mas COM os pés} APOIADOS NO CHÃO OU NUM BANQUINHO

INSTRUÇÕES: Por favor, fique sentado sem apoiar as costas com os braços cruzados por 2 minutos.

( ) 4 capaz de permanecer sentado com segurança e com firmeza por 2 minutos

( ) 3 capaz de permanecer sentado por 2 minutos sob supervisão

( ) 2 capaz de permanecer sentado por 30 segundos

( ) 1 capaz de permanecer sentado por 10 segundos

( ) 0 incapaz de permanecer sentado sem apoio durante 10 segundos

4. POSIÇÃo EM PÉ PARA POSIÇÃO SENTADA

INSTRUÇÕES: Por favor, sente-se.

( ) 4 senta-se com segurança com uso mínimo das mãos

( ) 3 controla a descida utilizando as mãos

( ) 2 utiliza a parte posterior das pernas contra a cadeira para controlar a descida

( ) 1 senta-se independentemente, mas tem descida sem controle

( ) 0 necessita de ajuda para sentar-se

\section{TRANSFERÊNCIAS}

INSTRUÇÕES: Arrume as cadeiras perpendicularmente ou uma de frente para a outra para uma transferência em pivô. Peça ao paciente para transferir-se de uma cadeira com apoio de braço para uma cadeira sem apoio de braço, e vice-versa. Você poderá utilizar duas cadeiras (uma com e outra sem apoio de braço) ou uma cama e uma cadeira.

( ) 4 capaz de transferir-se com segurança com uso mínimo das mãos

( ) 3 capaz de transferir-se com segurança com o uso das mãos

( ) 2 capaz de transferir-se seguindo orientações verbais e/ou supervisão

( ) 1 necessita de uma pessoa para ajudar

( ) 0 necessita de duas pessoas para ajudar ou supervisionar para realizar a tarefa com segurança

6. PERMANECER EM PÉ SEM APOIO COM OS OLHOS FECHADOS

INSTRUÇÕES: Por favor, fique em pé e feche os olhos por 10 segundos.

( ) 4 capaz de permanecer em pé por 10 segundos com segurança

( ) 3 capaz de permanecer em pé por 10 segundos com supervisão

( ) 2 capaz de permanecer em pé por 3 segundos

( ) 1 incapaz de permanecer com os olhos fechados durante 3 segundos, mas mantém-se em pé

( ) 0 necessita de ajuda para não cair

7. PERMANECER EM PÉ SEM APOIO COM OS PÉS JUNTOS

INSTRUÇÕES: Junte seus pés e fique em pé sem se apoiar.

( ) 4 capaz de posicionar os pés juntos independentemente e permanecer por 1 minuto com segurança

( ) 3 capaz de posicionar os pés juntos independentemente e permanecer por 1 minuto com supervisão

( ) 2 capaz de posicionar os pés juntos independentemente e permanecer por 30 segundos

( ) 1 necessita de ajuda para posicionar-se, mas é capaz de permanecer com os pés juntos durante 15 segundos

( ) 0 necessita de ajuda para posicionar-se e é incapaz de permanecer nessa posição por15 segundos 


\section{ALCANÇAR A FRENTE COM O BRAÇO ESTENDIDO PERMANECENDO EM PÉ}

INSTRUÇÕES: Levante o braço a $90^{\circ}$. Estique os dedos e tente alcançar a frente o mais longe possível.

( $O$ examinador posiciona a régua no fim da ponta dos dedos quando o braço estiver a $90^{\circ}$. Ao serem esticados para frente, os dedos não devem tocar a régua. A medida a ser registrada é a distância que os dedos conseguem alcançar quando o paciente se inclina para frente o máximo que ele consegue. Quando possível peça ao paciente para usar ambos os braços para evitar rotação do tronco).

( ) 4 pode avançar a frente $>25 \mathrm{~cm}$ com segurança

( ) 3 pode avançar a frente $>12,5 \mathrm{~cm}$ com segurança

( ) 2 pode avançar a frente $>5 \mathrm{~cm}$ com segurança

( ) 1 pode avançar a frente, mas necessita de supervisão

( ) 0 perde o equilíbrio na tentativa, ou necessita de apoio externo

\section{PEGAR UM OBJETO DO CHÃO A PARTIR DE UMA POSIÇÃO EM PÉ}

INSTRUÇÕES: Pegue o sapato/chinelo que está na frente dos seus pés.

( ) 4 capaz de pegar o chinelo com facilidade e segurança

( ) 3 capaz de pegar o chinelo, mas necessita de supervisão

( ) 2 incapaz de pegá-lo, mas se estica até ficar a $2-5 \mathrm{~cm}$ do chinelo e mantém o equilíbrio independentemente

( ) 1 incapaz de pegá-lo, necessitando de supervisão enquanto está tentando

( ) 0 incapaz de tentar, ou necessita de ajuda para não perder o equilíbrio ou cair

10. VIRAR-SE E OLHAR PARA TRÁS POR CIMA DOS OMBROS DIREITO E ESQUERDO ENQUANTO PERMANECE EM PÉ

INSTRUÇÕES: Vire-se para olhar diretamente atrás de você por cima do seu ombro esquerdo sem tirar os pés do chão. Faça o mesmo por cima do ombro direito.

(O examinador poderá pegar um objeto e posicioná-lo diretamente atrás do paciente para estimular o movimento).

( ) 4 olha para trás de ambos os lados com uma boa distribuição do peso

( ) 3 olha para trás somente de um lado, o lado contrário demonstra menor distribuição do peso

( ) 2 vira somente para os lados, mas mantém o equilíbrio

( ) 1 necessita de supervisão para virar

( ) 0 necessita de ajuda para não perder o equilíbrio ou cair

\section{GIRAR 360 GRAUS}

INSTRUÇÕES: Gire-se completamente ao redor de si mesmo. Pausa. Gire-se completamente ao redor de si mesmo em sentido contrário.

( ) 4 capaz de girar 360 graus com segurança em 4 segundos ou menos

( ) 3 capaz de girar 360 graus com segurança somente para um lado em 4 segundos ou menos

( ) 2 capaz de girar 360 graus com segurança, mas lentamente

( ) 1 necessita de supervisão próxima ou orientações verbais

( ) 0 necessita de ajuda enquanto gira 


\section{POSICIONAR OS PÉS ALTERNADAMENTE NO DEGRAU OU BANQUINHO} ENQUANTO PERMANECE EM PÉ SEM APOIO

INSTRUÇÕES: Toque cada pé alternadamente no degrau/banquinho. Continue até que cada pé tenha tocado o degrau/banquinho quatro vezes.

( ) 4 capaz de permanecer em pé independentemente e com segurança, completando 8 movimentos em 20 segundos

( ) 3 capaz de permanecer em pé independentemente e completar 8 movimentos em $>20$ segundos

( ) 2 capaz de completar 4 movimentos sem ajuda

( ) 1 capaz de completar >2 movimentos com o mínimo de ajuda

( ) 0 incapaz de tentar, ou necessita de ajuda para não cair

\section{PERMANECER EM PÉ SEM APOIO COM UM PÉ À FRENTE}

INSTRUÇÕES: (DEMONSTRE PARA O PACIENTE)

Coloque um pé diretamente à frente do outro na mesma linha, se você achar que não irá conseguir, coloque o pé um pouco mais à frente do outro pé e levemente para o lado.

( ) 4 capaz de colocar um pé imediatamente à frente do outro, independentemente, e permanecer por 30 segundos

( ) 3 capaz de colocar um pé um pouco mais à frente do outro e levemente para o lado, independentemente, e permanecer por 30 segundos

( ) 2 capaz de dar um pequeno passo, independentemente, e permanecer por 30 segundos

( ) 1 necessita de ajuda para dar o passo, porém permanece por 15 segundos

( ) 0 perde o equilíbrio ao tentar dar um passo ou ficar de pé

\section{PERMANECER EM PÉ SOBRe UMA PERNA}

INSTRUÇÕES: Fique em pé sobre uma perna o máximo que você puder sem se segurar.

( ) 4 capaz de levantar uma perna independentemente e permanecer por $>10$ segundos

( ) 3 capaz de levantar uma perna independentemente e permanecer por 5-10 segundos

( ) 2 capaz de levantar uma perna independentemente e permanecer por $\geq 3$ segundos

( ) 1 tenta levantar uma perna, mas é incapaz de permanecer por 3 segundos, embora permaneça em pé independentemente

( ) 0 incapaz de tentar, ou necessita de ajuda para não cair

( ) ESCORE TOTAL $($ Máximo $=56)$ 


\section{AVALIAÇÃO DO EQUILÍBRIO ESTÁTICO}

\section{Teste Clínico para Avaliação da Integração Sensorial no Equilíbrio} (CTSIB)

Permanecer em cada condição pelo menos 30 segundos.

\begin{tabular}{|c|c|c|c|c|c|}
\hline Condição 1 & Condição 2 & Condição 3 & Condição 4 & Condição 5 & Condição 6 \\
\hline _seg & _seg & _seg & _seg & _seg & \\
\hline
\end{tabular}

\section{AVALIAÇÃO DA MOBILIDADE FUNCIONAL}

Timed "Up \& Go" Test (TU\&GT)

Teste do Tempo de Ir e Vir

Cronometrar o tempo que uma pessoa leva para levantar-se de uma cadeira, caminhar três metros, dar uma volta, andar de volta e sentar-se novamente na cadeira.

Tempo gasto na tarefa: segundos 
ANEXO 5

\section{CARTILHA DE EXERCÍcIOS}

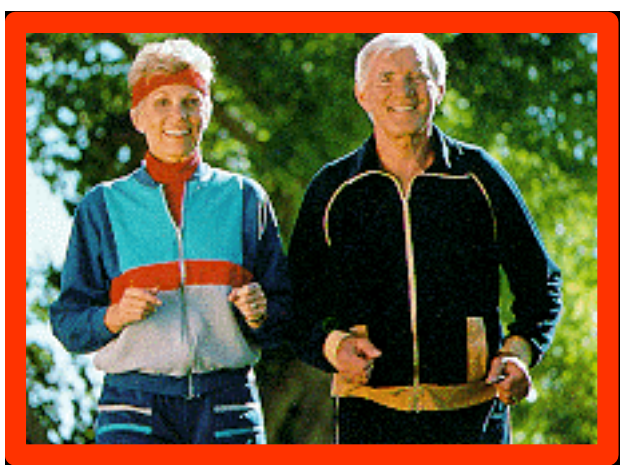

\section{PROGRAMA DE ORIENTAÇÃO À ATIVIDADE FÍSICA PARA PACIENTES COM OSTEOPOROSE}

Fisioterapeuta Responsável: Melisa Moreira Madureira

Reumatologista: Profa. Dra. Rosa Maria Rodrigues Pereira Titular da Disciplina de Reumatologia: Profa. Dra. Eloisa Bonfá

Faculdade de Medicina da Universidade de São Paulo Laboratório de Metabolismo Ósseo - LIM 17 - Departamento de Reumatologia 


\section{SUMÁRIO}

O QUE É OSTEOPOROSE?

FATORES DE RISCO PARA OSTEOPOROSE

DIAGNÓSTICO E PREVENÇÃO DA OSTEOPOROSE

RECOMENDAÇÕES PARA REALIZAR ATIVIDADE FÍSICA

TREINO DE EQUILÍBRIO E COORDENAÇÃO MOTORA

ALONGAMENTOS

FORTALECIMENTO MUSCULAR

EXERCÍCIOS CONTRA-INDICADOS NA OSTEPOROSE

DICAS DE POSTURA PARA FAZER AS ATIVIDADES DIÁRIAS

A PREVENÇÃO DE QUEDAS É FUNDAMENTAL PARA EVITAR FRATURAS

A IMPORTÂNCIA DA DIETA NA OSTEOPOROSE 


\section{O QUE É OSTEOPOROSE?}

Osteoporose é uma doença que pode atingir todos os ossos do corpo, fazendo com que fiquem fracos aumentando a possibilidade de se quebrarem aos mínimos esforços.

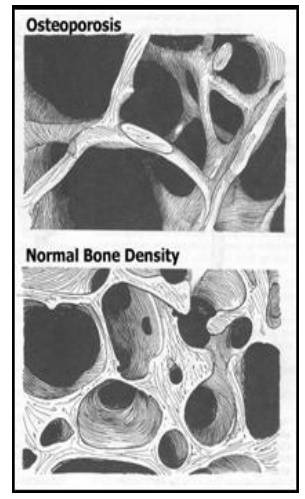

Osso com osteoporose

Osso normal

\section{Os principais tipos de osteoporose são:}

$\checkmark$ Osteoporose pós-menopausa: atinge mulheres após a menopausa, fratura de coluna pode ocorrer.

$\checkmark \quad$ Osteoporose senil: atinge pessoas com mais de 70 anos. Tanto a fratura de coluna quanto à de quadril podem ocorrer.

$\checkmark$ Osteoporose secundária: atinge pessoas com doença renal hepática, endócrina, hematológica, ou que usam alguns medicamentos, por exemplo, corticóides. 


\section{FATORES DE RISCO PARA OSTEOPOROSE}

Há um conjunto de fatores que influenciam e favorecem o desenvolvimento da Osteoporose.

$\checkmark$ Envelhecimento: a perda de massa óssea aumenta com a idade.

$\checkmark$ Menopausa: com a interrupção da menstruação ocorre diminuição dos níveis de estrógenos (hormônio feminino), que é fundamental para manter a massa óssea.

$\checkmark$ Dieta pobre em cálcio: o cálcio é fundamental na formação óssea. Sua obtenção a partir da alimentação é imprescindível para se prevenir a osteoporose.

$\checkmark$ Hereditariedade: a osteoporose é mais freqüente em pessoas com antecedentes familiares da doença.

$\checkmark$ Imobilização prolongada: o exercício físico constitui um importante estímulo para a formação e fortalecimento dos ossos. Grandes períodos de imobilização e a falta de exercícios podem levar à osteoporose.

$\checkmark$ Medicamentos: alguns medicamentos, como os corticóides, em tratamentos de longa duração, favorecem a destruição óssea.

$\checkmark$ Excesso de fumo e álcool: tem-se observado uma maior incidência de osteoporose entre as pessoas que consomem álcool e fumo em excesso. 


\section{Pode-se suspeitar ou prever a osteoporose?}

A resposta é afirmativa para pessoas que se enquadram nestas circunstâncias:

$\checkmark$ Pessoas com mais de 60 anos

$\checkmark$ Mulheres pós-menopausa

$\checkmark$ Com antecedentes familiares

$\checkmark$ Dieta pobre em cálcio

$\checkmark$ Fumantes e consumidores de álcool

$\checkmark$ Pessoas sedentárias

$\checkmark$ Em tratamento prolongado com corticóides

A osteoporose pode não provocar sintomas. A dor pode acontecer em conseqüência de fratura, que pode ocorrer espontaneamente principalmente em coluna, quadril e punho. O osso fica tão fraco que pode haver achatamento da vértebra. Pode ocorrer encurvamento da coluna ("corcunda") e diminuição da altura.

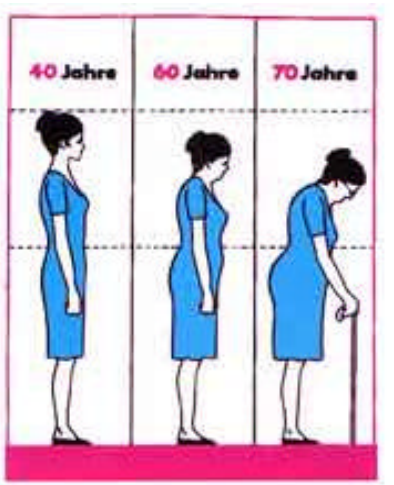




\section{DIAGNÓSTICO E PREVENÇÃO DA OSTEOPOROSE}

O exame mais adequado para o diagnóstico da osteoporose é a densitometria óssea. Esse exame permite avaliar o estágio da doença e serve como método de acompanhamento do tratamento. É um exame indolor que mede a massa óssea na coluna e fêmur.

\section{A osteoporose pode ser prevenida?}

Sim. A prevenção engloba uma série de medidas:

$\checkmark$ Nutrição adequada: dieta rica em leite e derivados.

$\checkmark$ Exercícios suaves: desde caminhadas até realização de um programa de exercícios estabelecido pelo médico ou pelo fisioterapeuta.

$\checkmark$ Parar de fumar

$\checkmark$ Evitar excesso de álcool

$\checkmark$ Também não se deve abusar do café e dieta com muito sal.

Tratamento medicamentoso (Deve ser indicado e prescrito pelo médico):

$\checkmark$ Cálcio

$\checkmark$ Vitamina D

$\checkmark$ Raloxifeno

$\checkmark$ Bisfosfonatos: Alendronato, Risendronato, Ibandronato 
$\checkmark$ Calcitonina

$\checkmark$ PTH

$\checkmark$ Ranelato de Estrôncio

\section{RECOMENDAÇÕES PARA REALIZAR ATIVIDADE FÍSICA}

Qualquer programa de atividade física deverá ser precedido de uma avaliação médica;

$\checkmark$ Realizar exercício somente quando houver bem estar físico;

$\checkmark$ Usar roupas e calçados adequados, de preferência tênis;

$\checkmark$ Evitar o fumo e o uso de sedativos;

$\checkmark$ Não se exercitar em jejum;

$\checkmark$ Dar preferência aos carboidratos antes do exercício;

$\checkmark$ Respeitar os limites pessoais, interrompendo o exercício se houver dor ou desconforto;

$\checkmark$ Evitar extremos de temperatura e umidade;

$\checkmark$ Iniciar a atividade física lenta e gradativamente para permitir a adaptação do corpo;

$\checkmark$ Hidratação adequada do organismo antes, durante e depois da atividade física. 


\section{TREINO DE EQUILÍBRIO E COORDENAÇÃO MOTORA}

1. Fique em um pé só:

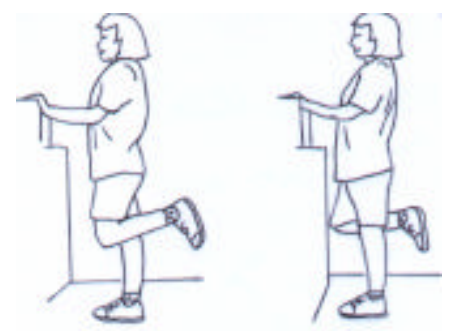

Inicialmente com apoio (gradualmente, aumente o tempo e retire as mãos do apoio).

2. Ande com um pé na frente do outro:
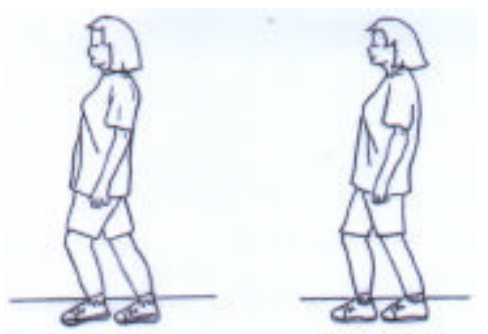

3. Ande apoiando-se no calcanhar:
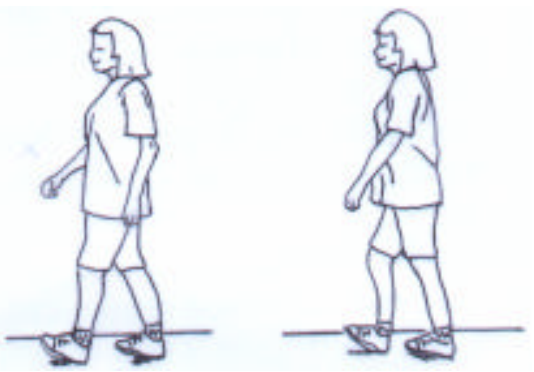
4. Ande levantando a perna:
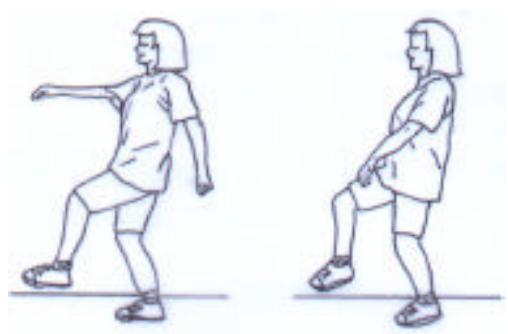

5. Ande de lado:
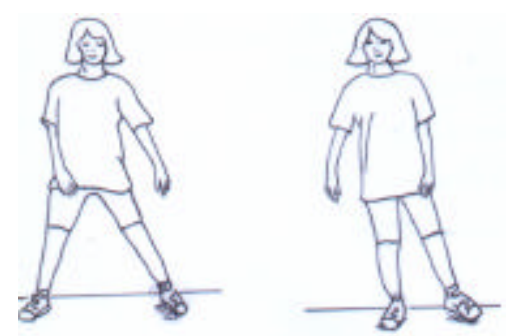

6. Ande levantando uma perna e o braço contralateral ao mesmo tempo:
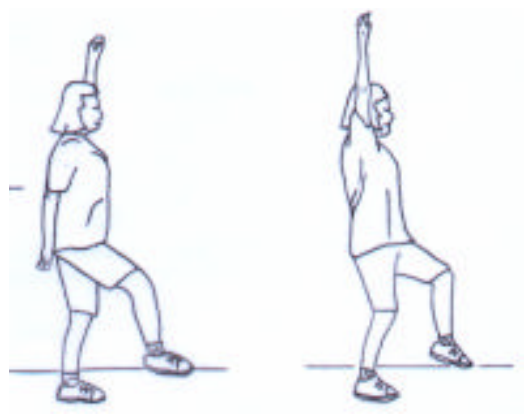

\section{Caminhada:}

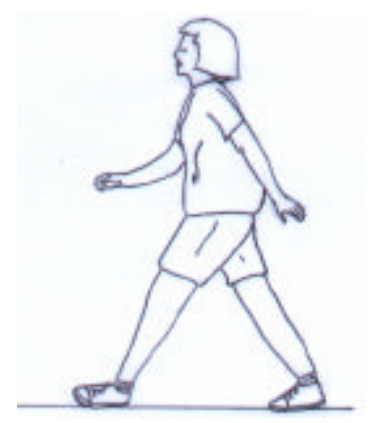

A caminhada é essencial 4 vezes por semana durante 40 minutos.

Durante a caminhada, endireite o seu corpo e ande o mais ereto possível, com o queixo para dentro e a cabeça bem levantada.

A caminhada se revela altamente efetiva no controle da postura, uma simples caminhada é a arma mais poderosa no combate ao desequilíbrio. 


\section{ALONGAMENTOS}

\section{Pescoço}

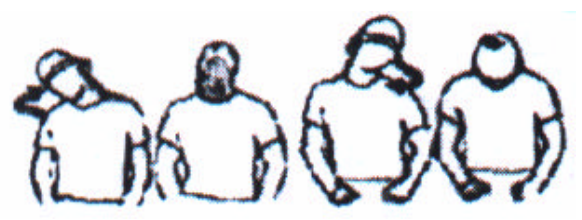

Incline a cabeça em direção ao ombro, para o lado direito e esquerdo, para cima e para baixo.

\section{Tríceps}

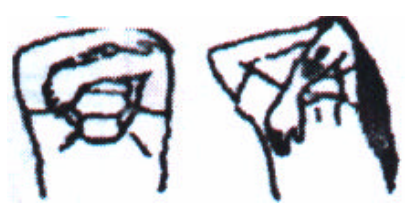

Coloque a mão atrás da cabeça e, com a mão contralateral, puxe o cotovelo para baixo.

\section{Músculo peitoral:}

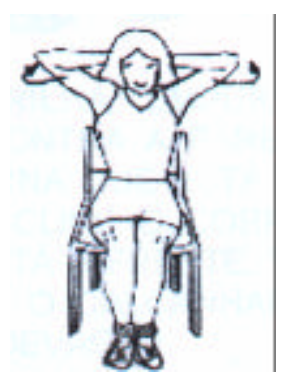

Cruze as mãos atrás da cabeça e empurre os cotovelos para trás abrindo o peito (estimule a respiração profunda). 


\section{Músculos para-vertebrais e glúteos:}

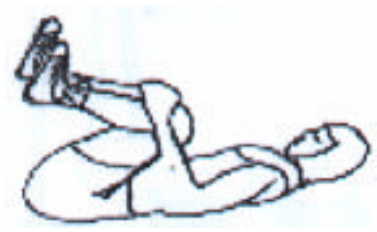

Aperte com as mãos os joelhos e, ao mesmo tempo, force as coxas e joelhos em direção ao tórax. Conte até 10, devagar, e depois solte. Certifique-se de que os ombros e o pescoço estão relaxados.

\section{Panturrilha:}

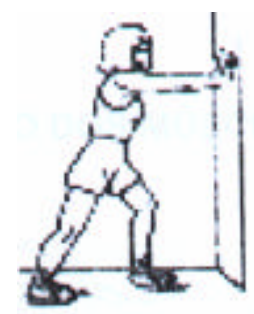

Empurre a parede (alterne os pés). Coloque as mãos contra a parede com uma perna atrás da outra. Mantenha a perna que está reta e os dedos olhando na direção da parede. Incline o corpo para frente, devagar, dobrando a perna que está à frente, você deve sentir alongar a panturrilha, sem tirar o calcanhar do chão. Segure, nesta posição, contando até 10 devagar. 


\section{6. Ísquios tibiais (musculatura posterior da coxa):}

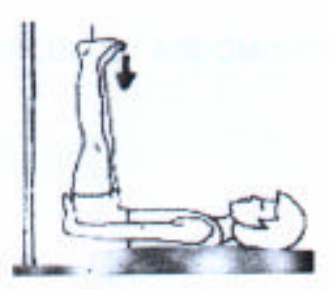

Com as pernas apoiadas na parede, force os dedos dos pés na direção do seu corpo. Conte até 10, devagar, e descanse.

\section{Músculo quadríceps}

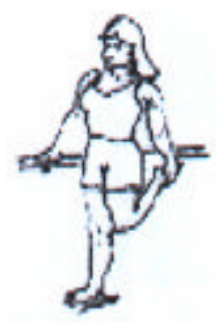

Apóie uma mão na parede, dobre o joelho para trás elevando o pé e puxe-o com a mão em direção ao glúteo.

\section{FORTALECIMENTO MUSCULAR}

\section{Músculos abdominais:}

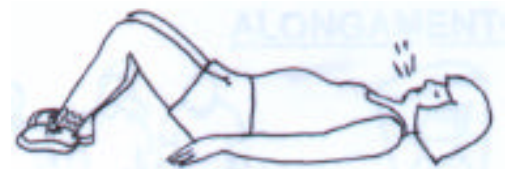

Respire pelo nariz, solte 0 ar pela boca: coloque as mãos na barriga, respire jogando o ar para a barriga (encha a barriga) e depois fazendo bico, solte 0 ar, devagar, até acabar 0 ar dos pulmões e sinta a barriga endurecer. Não flexionar a coluna. 


\section{Membros superiores:}

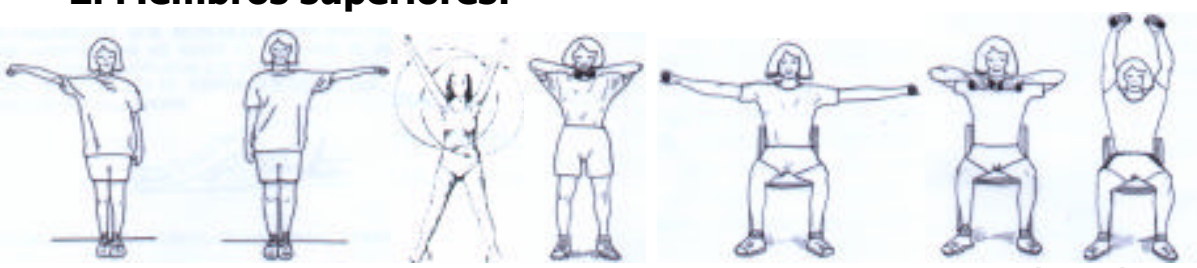

Os exercícios são: Levantar os braços lateralmente, até o nível dos ombros;. Realizar movimentos em círculos com os braços; imitar boxe; rodar os ombros e estendê-los; rodar os braços e os punhos (progredindo-se acrescentar pesos); Pés afastados (bater palma na frente e para cima).

\section{Musculatura extensora da coluna:}

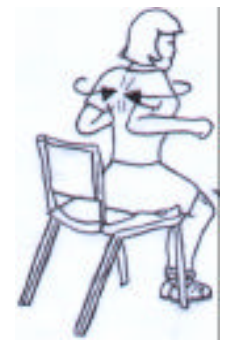

a) Sem apoiar-se no encosto da cadeira, dobre os cotovelos e tente juntá-los atrás das costas. Conte até 10 e relaxe.

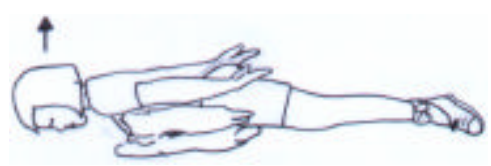

b) Deite de bruços, com uma almofada debaixo do tórax e abdômen, com os braços estendidos por trás das costas e com as pernas esticadas, apoiando os dedos dos pés no chão.

\section{Abdutores (quadril):}

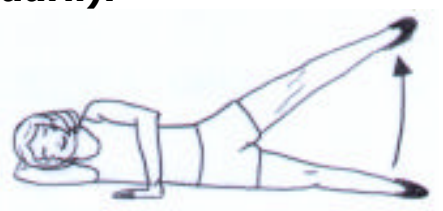

Deite-se de lado, force os dedos na direção do seu corpo, esticando o joelho. Levante a perna e conte até 10 . Apóie a perna novamente, mantendo-a esticada. 


\section{Glúteos:}

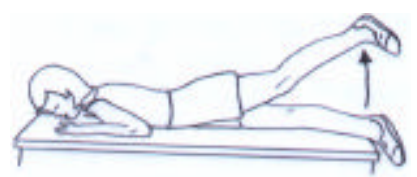

Deite-se de barriga para baixo, esticando o joelho. Levante a perna, conte até 10 e relaxe.

\section{Quadríceps (coxa):}

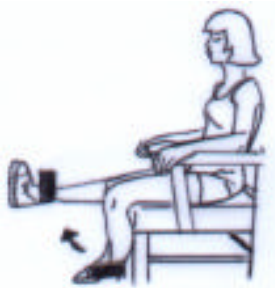

Sentada em uma cadeira, apoiando os braços, levante o dedão do pé e o tornozelo, estique o joelho, conte até 10 , descanse e repita na outra perna (progredindo, acrescente peso gradualmente).

\section{EXERCÍCIOS CONTRA-INDICADOS NA OSTEPOROSE}

\section{Evite movimentos de flexão e torção da coluna}

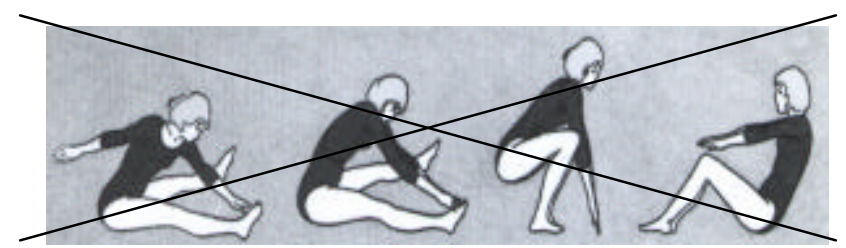



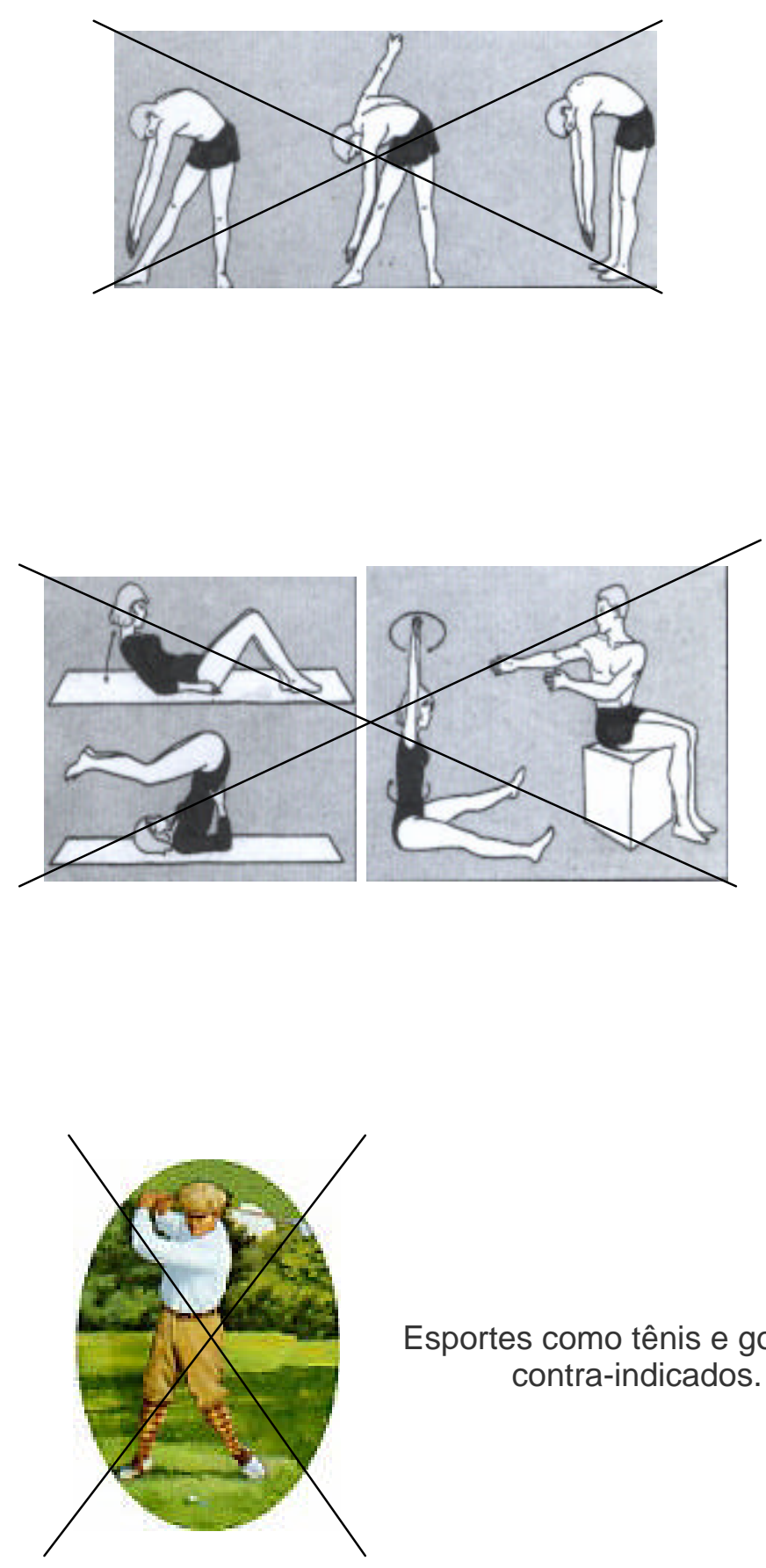

Esportes como tênis e golfe são contra-indicados. 


\section{DICAS DE POSTURA PARA FAZER AS ATIVIDADES DIÁRIAS}

\section{Lavar louça ou roupa:}

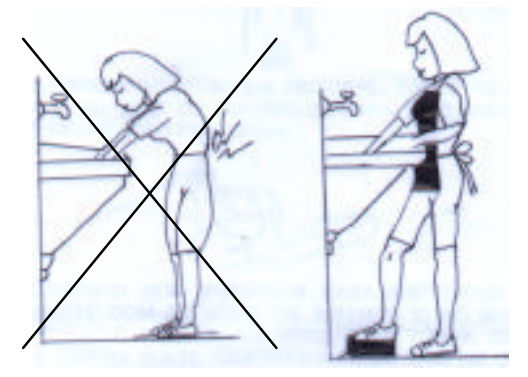

Apóie o pé dentro do gabinete da cozinha, retificando a coluna. Use avental e encoste bem o corpo no tanque ou na pia.

\section{Passar roupa:}

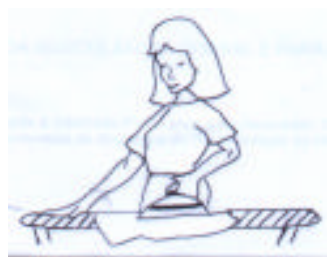

Apóie um dos pés em uma lista telefônica, banquinho ou tijolo e passe de lado. 
3. Aumentar o cabo da vassoura (evitando dobrar a coluna):

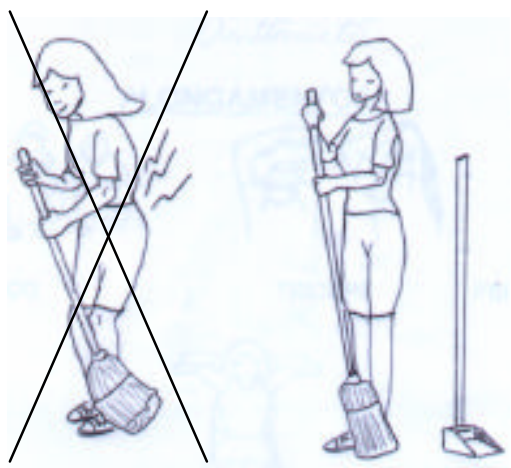

4. Distribuir o peso em duas sacolas:
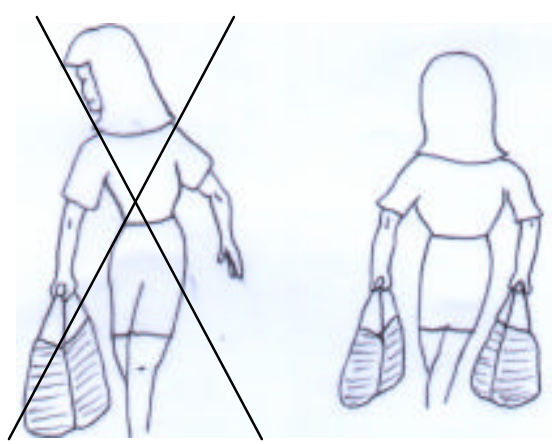

Não carregue coisas pesadas. Use carrinho para transportar objetos pesados.

\section{Sapatos:}
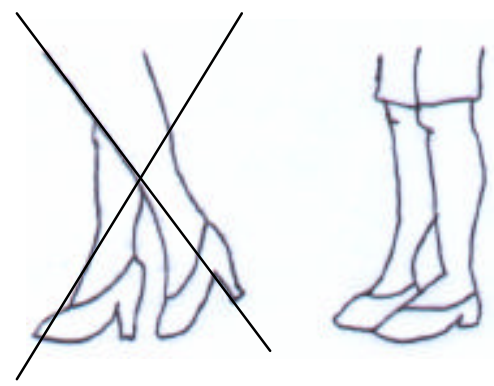

Usar sapatos com bico quadrado e salto de 1 a $2 \mathrm{~cm}$. Ao invés de saltos ou sapatos com bico fino. 


\section{Para se vestir ou calçar:}
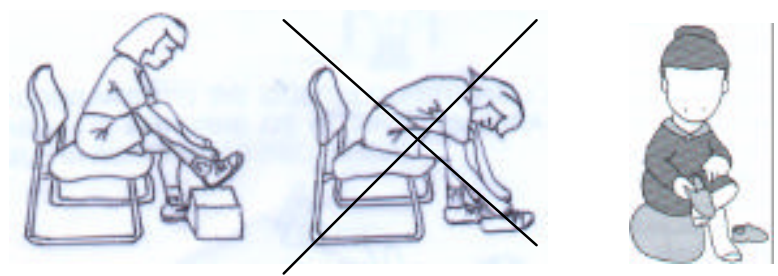

Para vestir a calça, meia ou calçar sapatos, permaneça sentado. Para amarrar os sapatos, traga o pé de encontro ao corpo e nunca a coluna em direção ao pé. Para amarrar os sapatos, cortar as unhas ou secar os pés sente-se em uma cadeira e coloque um dos pés sobre um apoio ou caixa, mantendo o alinhamento adequado das costas.

\section{Tossir ou espirrar:}

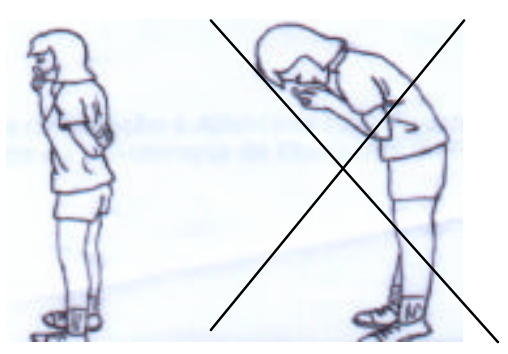

Desenvolva o hábito de sustentar as costas. Isso protege a coluna e os discos intervertebrais das lesões causadas por movimentos repentinos. 


\section{Pegar objetos do chão:}

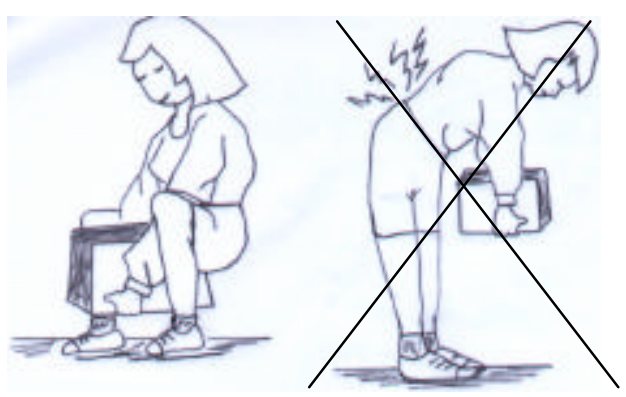

Ao levantar um objeto do chão, não dobre as costas, dobre os joelhos. Procure apoiar um dos joelhos no chão. Mantenha-se nessa posição pelo menor tempo possível, levante o objeto bem junto ao corpo, próximo da cintura e não carregue coisas muitas pesadas.

\section{Deitar-se:}

a) O colchão deve ser firme de boa qualidade e confortável. Ao deitarse de lado, o travesseiro deve ter a altura do ombro.

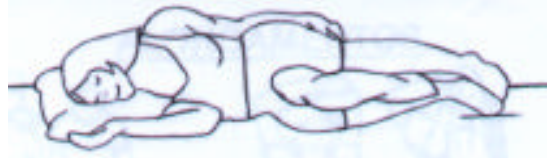


b) Ao dormir, deite-se de lado, com um travesseiro entre as pernas (para distribuir o peso melhor sobre a coluna). Deite com os joelhos esticados e nunca coloque o travesseiro abaixo dos joelhos.

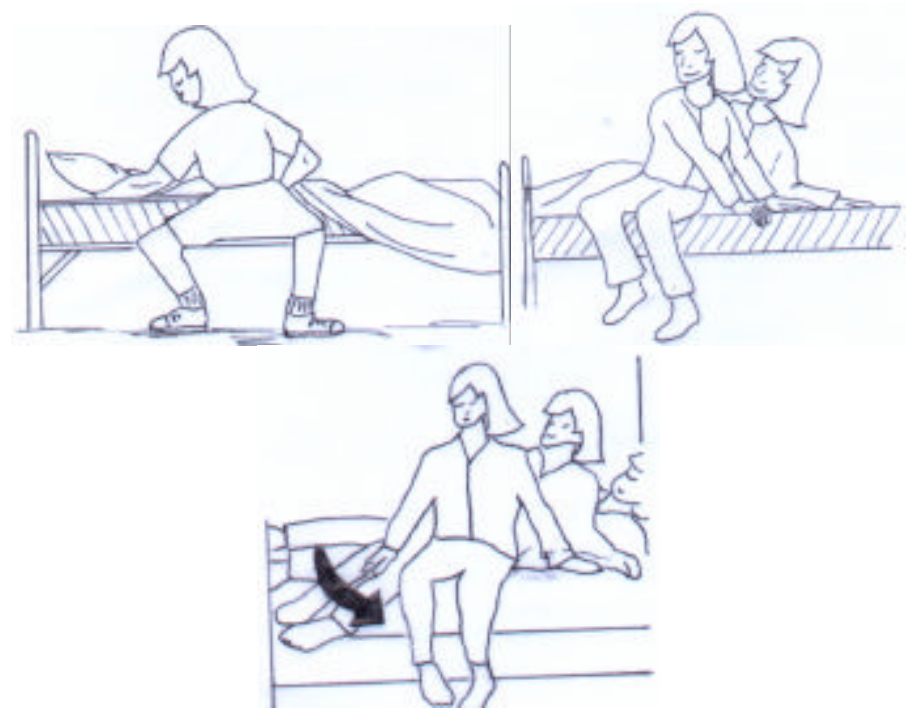

c) Para levantar-se da cama, primeiro fique de lado, tire as pernas para fora do colchão e erga o corpo usando o cotovelo e a mão como apoios na cama. Quando já estiver sentado, vá para a beirada da cama e somente, quando estiver com os pés apoiados no chão e não sentir tontura, levante-se com cuidado. Faça o mesmo para deitar, apóie o cotovelo, as mãos e deite primeiro de lado. 


\section{A PREVENÇÃO DE QUEDAS É FUNDAMENTAL PARA EVITAR FRATURAS}

$\checkmark$ Não deixe fios de telefone e televisão expostos ou soltos no chão.

$\checkmark$ Não deixe animais e objetos espalhados pelo chão.

$\checkmark$ Não deixe tapetes soltos.

$\checkmark$ Evite calçado de saltos e com solado liso, use sapatos confortáveis com solado antiderrapante.

$\checkmark$ Não encere o assoalho, pois isso torna o chão escorregadio.

$\checkmark$ Não ande em locais pouco iluminados e com chão molhado.

$\checkmark$ Utilize corrimão dos dois lados, não coloque tapetes nas escadas.

$\checkmark$ Não guarde objetos em prateleiras altas.

$\checkmark$ Coloque banco de plástico dentro do boxe para, sentado, lavar os pés e use ducha móvel. Coloque barras de segurança no banheiro. Se possível tenha piso antiderrapante na cozinha e no banheiro.

$\checkmark$ Coloque corrimões e barras de apoios próximos à cama, ao vaso sanitário e dentro do boxe do banheiro.

$\checkmark$ Quando acordar à noite, espere alguns minutos antes de se levantar para ir ao banheiro ou tomar água, principalmente, se estiver usando remédio que provoque tontura.

$\checkmark$ À noite, tenha sempre uma lanterna no criado-mudo.

Uma boa visão é fundamental para o bom equilíbrio, portanto, visite com freqüência seu oftalmologista. 


\section{A IMPORTÂNCIA DA DIETA NA OSTEOPOROSE}

A nutrição adequada exerce grande influência na prevenção da osteoporose, daí a importância de uma alimentação balanceada, obtida através dos alimentos, que são divididos em três grupos:

Alimentos energéticos: fornecem energia ao nosso organismo para todas as atividades diárias, como: andar, se alimentar, trabalhar e até mesmo dormir. São eles: carboidratos (massas, pães, arroz, batata, inhame, mandioca e mandioquinha) e gorduras (óleos, margarina e manteiga).

Alimentos construtores: são responsáveis pela formação e constante renovação de tecidos do nosso organismo, como crescimento de cabelos e unhas, cicatrização de feridas. São eles: proteínas (carnes, leite, queijos e grãos).

Alimentos reguladores: regulam todas as funções do nosso organismo. São eles: vitaminas e sais minerais (encontrados em legumes, verduras e frutas). 
A pirâmide abaixo mostra os grupos de alimentos e o número de porções que deve ser consumido de cada um deles:

Gorduras e açúcares: 1-2 porções

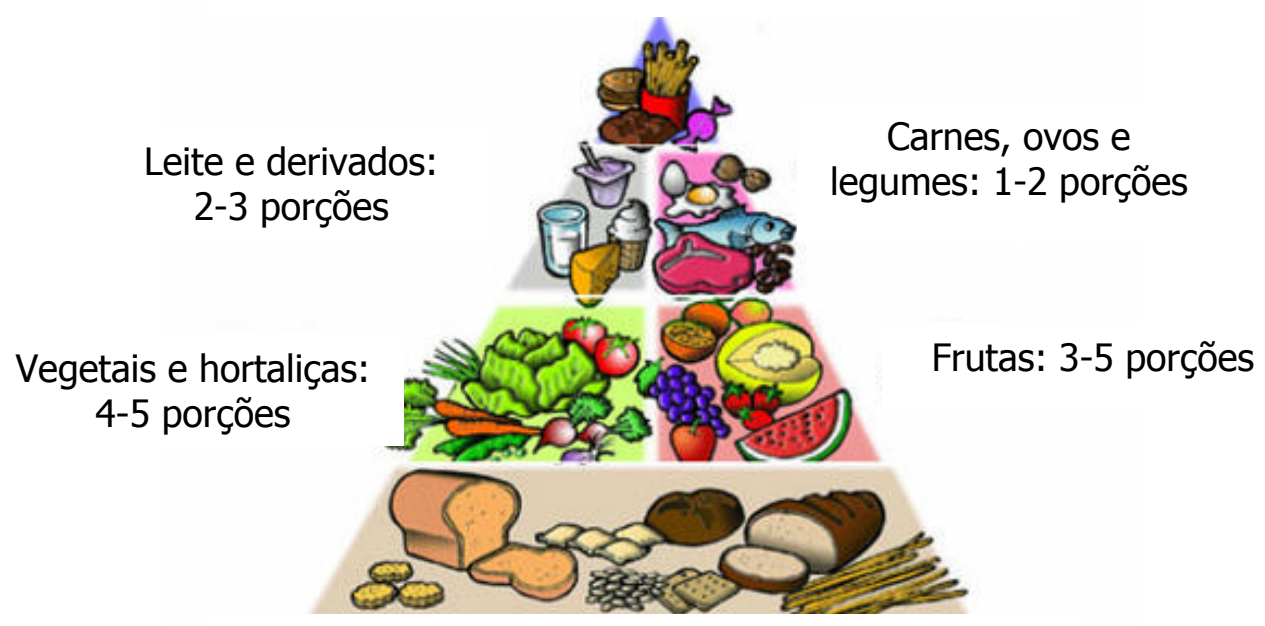

Cereais, pães, massas e raízes: 5-9 porções

Além disso, existe um nutriente imprescindível na prevenção da osteoporose, que é o cálcio, já que cerca de 99\% dele encontra-se nos ossos e dentes, sendo um mineral fundamental para formação e manutenção óssea.

Com o passar da idade, ocorre diminuição na absorção de cálcio e aumento da eliminação do mesmo. Sua ingestão inadequada pode resultar em redução da massa óssea, principalmente, após os 50 anos, em ambos os sexos, progredindo mais rapidamente nas mulheres.

Sendo assim, é importante uma adequada oferta de cálcio proveniente da alimentação desde a infância, em que ocorre a formação dos hábitos alimentares, até a fase adulta. 
A recomendação de cálcio, segundo a RDA (Recommend Diary Allowed), é de $1.200 \mathrm{mg} /$ dia para adultos e de $1.500 \mathrm{mg} /$ dia para mulheres no período pós-menopausa.

Segue abaixo uma tabela com os principais alimentos ricos em cálcio, bem como a quantidade deste mineral na porção recomendada:

\begin{tabular}{|c|c|c|}
\hline ALIMENTOS & QUANTIDADE & CÁLCIO (mg) \\
\hline Leite integral & 1 copo $(200 \mathrm{~mL})$ & 228 \\
\hline Leite desnatado & 1 copo $(200 \mathrm{~mL})$ & 246 \\
\hline Leite de soja & 1 copo $(200 \mathrm{~mL})$ & 80 \\
\hline Leite de cabra & 1 copo $(200 \mathrm{ml})$ & 380 \\
\hline Queijo minas fresco & 1 fatia $(m)-30 g$ & 205 \\
\hline Queijo prato & 1 fatia fina- $15 \mathrm{~g}$ & 126 \\
\hline Queijo parmesão & 1 colher sob.-10 mg & 114 \\
\hline Requeijão & 1 porção-20g & 113 \\
\hline Iorgute & 1 pote $(200 \mathrm{~mL})$ & 240 \\
\hline Espinafre & 2 c. sopa- $60 \mathrm{~g}$ & 47 \\
\hline Couve-manteiga & 3 c. sopa- $36 g$ & 73 \\
\hline Escarola & 3 c. sopa-36g & 29 \\
\hline Agrião & 1 prato sob.-20g & 24 \\
\hline Brócolis & 3 c. sopa-36g & 37 \\
\hline Sardinha & 1 porção-30g & 86 \\
\hline Ostras & 1 porção- $240 \mathrm{~g}$ & 235 \\
\hline
\end{tabular}


Portanto, para se atingir a quantidade de cálcio recomendada por dia em adultos, é necessário, por exemplo, a ingestão de 1 copo de leite desnatado com duas fatias de queijo minas pela manhã, 1 iorgute no lanche da tarde com 2 porções de requeijão e 1 copo de leite à noite, antes de dormir, sendo os demais alimentos consumidos normalmente.

Uma boa tática é acrescentar leite em pó, preferencialmente, desnatado no leite, aumentando assim a quantidade total de cálcio ingerido.

Além disso, uma adequada ingestão de alimentos ricos em vitamina $D$ também auxilia na prevenção e no tratamento da osteoporose, já que esta melhora o aproveitamento do cálcio ingerido.

Os alimentos ricos em vitamina $D$ são: leite e produtos lácteos enriquecidos com vitamina $D$, óleo de fígado de peixe (bacalhau, sardinha, salmão e atum), ostras, camarão, peixes (salmão, atum, sardinha) e ovos.

Entretanto, a principal fonte de vitamina D é obtida através da luz solar, sendo necessária uma exposição a ela de cerca de 15 minutos/dia, preferencialmente, nos horários da manhã (até às 10h) e à tarde (após as 15h).

\section{Alimentos prejudiciais à osteoporose:}

Evite alimentos como café, chás escuros, bebidas alcoólicas e dietas com quantidade excessiva de fibras, pois estas diminuem o aproveitamento do cálcio quando consumidas de forma exagerada. 
Existem também alguns alimentos que são inibidores do cálcio, como por exemplo:

$\checkmark$ Gorduras da dieta: formam sabões insolúveis com o cálcio da dieta, aumentando sua eliminação pelas fezes e diminuindo o seu aproveitamento;

$\checkmark$ Ácido oxálico: presente em vegetais (beterraba, semente de tomate, aspargo) e no cacau, chocolate, gérmen de trigo, nozes e feijão, forma complexos com cálcio, sendo eliminados pelas fezes;

$\checkmark$ Refrigerante do tipo cola possuem o ácido fosfórico que prejudica a formação óssea.

$\checkmark$ Dieta rica em sal - aumenta a excreção de cálcio pela urina

Portanto, lembre-se sempre:

"Procure manter alimentos ricos em cálcio em sua alimentação, colaborando para prevenir e tratar a osteoporose". 
ANEXO 6

CARTA DE ACEITE DO TRABALHO PARA REVISTA INDEXADA: Osteoporosis International (impacto 4,2).

De: oi.europe@lyon.inserm.fr

Para: rosamariarp@yahoo.com

Data: 06/10/2006 06:54

Assunto: Osteoporos Int - Decision on Manuscript ID OI-2006-06-0223.R1

\section{Mensagem}

06-Oct-2006

Manuscript ID: OI-2006-06-0223.R1

Dear Prof. Pereira:

It is a pleasure to accept your manuscript entitled "Balance Training Program is Highly Effective in Improving Functional Status and Reducing the Risk of Falls in Elderly Women with Osteoporosis: a Randomized Controlled Trial.," in its current form for publication in Osteoporosis International.

Could you please complete the attached Copyright Transfer and Authorship form and return it by fax to +33 472369052 .

Thank you for your fine contribution. On behalf of the Editors of Osteoporosis International, we look forward to your continued contributions to the Journal.

Yours sincerely, Ego Seeman

Editor, Osteoporosis International 
ANEXO 7

TRABALHO ACEITO PARA PUBLICAÇÃO EM REVISTA INDEXADA: Osteoporosis International (impacto 4,2).

"Balance Training Program is Highly Effective in Improving Functional Status and Reducing the Risk of Falls in Elderly Women with Osteoporosis: a Randomized Controlled Trial" 


\title{
Balance training program is highly effective in improving functional status and reducing the risk of falls in elderly women with osteoporosis: a randomized controlled trial
}

\author{
M. M. Madureira • L. Takayama • A. L. Gallinaro • \\ V. F. Caparbo • R. A. Costa - R. M. R. Pereira
}

Received: 9 June 2006 / Accepted: 6 October 2006

(C) International Osteoporosis Foundation and National Osteoporosis Foundation 2006

\begin{abstract}
Introduction The purpose of this study was to investigate the effect of a 12-month Balance Training Program on balance, mobility and falling frequency in women with osteoporosis.

Methods Sixty-six consecutive elderly women were selected from the Osteometabolic Disease Outpatient Clinic and randomized into 2 groups: the 'Intervention', submitted for balance training; and the 'Control', without intervention. Balance, mobility and falling frequency were evaluated before and at the end of the trial, using the Berg Balance Scale (BBS), the Clinical Test Sensory Interaction Balance (CTSIB) and the Timed "Up \& Go" Test (TUGT). Intervention used techniques to improve balance consisting of a 1-hour session each week and a home-based exercise program.

Results Sixty women completed the study and were analyzed. The BBS difference was significant higher in the Intervention group compared to Control $(5.5 \pm 5.67$ vs $-0.5 \pm 4.88$ score, $p<0.001)$. Similarly, the number of patients in the Intervention group presented improvement in two conditions of CTSIB compared to Control (eyes closed and unstable surface condition: 13 vs one patient, $p<0.001$ and eyes open, visual conflict and unstable surface condition: 12 vs one patient, $p<0.001$ ). Additionally, the
\end{abstract}

M. M. Madureira • L. Takayama • A. L. Gallinaro •

V. F. Caparbo · R. A. Costa $\cdot$ R. M. R. Pereira

Division of Rheumatology (Bone Mineral Metabolism

Laboratory), School of Medicine, University of São Paulo,

São Paulo, Brazil

R. M. R. Pereira $(\square)$

Faculdade de Medicina da Universidade de São Paulo, Av. Dr. Arnaldo, 455 - $3^{\circ}$ andar - Reumatologia, sala 3107, São Paulo, SP 01246-000, Brazil

e-mail: rosamariarp@yahoo.com differences between the TUGT were reduced in the Intervention group compared to Control $(-3.65 \pm 3.61$ vs $2.27 \pm 7.18$ seconds, $p<0.001)$. Notably, this improvement was paralleled by a reduction in the number of falls/patient in the Intervention group compared to Control $(-0.77 \pm 1.76$ vs $0.33 \pm 0.96, p=0.018)$.

Conclusion This longitudinal prospective study demonstrated that an intervention using balance training is effective in improving functional and static balance, mobility and falling frequency in elderly women with osteoporosis.

Keywords Balance $\cdot$ Exercise program $\cdot$ Falls $\cdot$ Mobility Osteoporosis

\section{Introduction}

Osteoporosis is a debilitating, widespread disease, which affects approximately $55 \%$ of the population above 50 years old in the USA [1]. Falls among the elderly, especially for those with osteoporosis, are associated with high morbidity and mortality and can involve high-cost medical intervention [2]. In fact, falls are responsible for $90 \%$ of the growing increase in hip fractures [3] and are the sixth cause of death among patients aged over 65 [4].

Falls are multifactorial, and their causes are categorized as intrinsic (personal) and extrinsic (environmental) factors $[5,6]$. Some examples of intrinsic factors include: altered balance, neurological diseases, sensory deterioration, musculoskeletal diseases, postural hypertension and the use of medication [7].

Research shows that altered balance is the greatest collaborator towards falls in the elderly [6, 8-12], with a 
high correlation between balance deficit and the incidence of falls $[13,14]$.

For this reason, studies regarding the risk of falling in osteoporosis are of high priority in clinical intervention. Diminishing the incidence of falls is a health priority, which reflects on both the quality of life and health costs [2].

Moreover, evidence suggests that exercise reduces the risk of fractures, showing an effect on the maintenance of bone mass and, more importantly, improving postural stability, mobility and, consequently, diminishing the risk of falls [15]. Indeed, improving balance should be an objective in the prevention of falls [16].

However, knowledge regarding balance deficit and the probability of falls is limited and controversial [17]. The literature shows that exercise may or may not be efficient in the control of falls [18], and the impact of prevention programs with balance training in diminishing falling frequency has yet to be established, principally in women with osteoporosis.

Balance training has been investigated in healthy elderly individuals [3, 16, 19-21]; however, only one study regarding balance training in women with osteoporosis exists. Since patients with osteoporosis are at greater risk of fractures resulting from falls, further research in this group should be prioritized [22].

The purpose of the present study was to investigate the effect of a 12-month balance training program on functional and static balance, mobility and falling frequency in women with osteoporosis.

\section{Patients and methods}

Patient sample

Sixty-six elderly women aged over 65 years old were consecutively selected from patients of the Osteometabolic Disease Outpatient Clinic of the Rheumatology Division, University of São Paulo. Only patients with osteoporosis, classified according to the World Health Organization [23] were included; with a bone mineral density (BMD) T-score lower than -2.5 standard deviation (SD), in the lumbar spine, femoral neck or total femur region.

The following women were excluded: those with secondary osteoporosis, visual deficiency, severe auditive deficiency, or vestibular alteration of important clinical status, such as women who used assisted walking devices or who were unable to walk independently more than 10 meters [24]; those who planned to be out of town for more than 4 weeks during the 12-month study; and women who presented absolute or relative contraindications for exercise training according to the American College of Sports Medicine [25].
The patients were randomized consecutively into two groups: the group submitted for the Balance Training Program (Intervention Group), consisting of 34 patients; and the Control group, consisting of 32 patients without intervention. The Control group only received treatment for osteoporosis and orientation to prevent falls and return regularly (3-monthly follow-ups) to the Osteometabolic Disease Outpatient Clinic. All patients read and signed a term of free informed consent that described the procedures which would be realized during the research.

Measured variables: interview and medical chart records

Personal, family and clinical data were evaluated through an interview and medical chart records, with emphasis on the history of fractures, number of falls in the preceding year, use of medication for osteoporosis, and use of medication that favored the risk of falling, such as hypnotics, hypotensors and antidepressants.

Functional state evaluation

Static and dynamic balance and mobility were evaluated in all patients, before and at the end of the trial, by a physiotherapist who was blinded to the distinct group (Intervention, Control).

\section{Functional balance}

The Berg Balance Scale (BBS) is based on 14 items common to daily life activities used to evaluate functional balance [26]. The maximum score that can be achieved is 56 , and each item possesses an ordinal scale of five alternatives which varies from 0 to 4 points.

The test is simple, easy to administer and accompanies the evolution of elderly patients. It only requires a ruler and a watch and takes approximately 15 minutes to execute [26]. A score lower than or equal to 45 is considered evidence of altered balance [27].

\section{Static balance}

Static balance was evaluated by the Clinical Test of Sensory Interaction for Balance (CTSIB), which consists of six sensory conditions (1: eyes open and firm surface, 2: eyes closed and firm surface, 3: eyes open, visual conflict and firm surface, 4: eyes open and unstable surface, 5: eyes closed and unstable surface, and 6: eyes open, visual conflict and unstable surface).

Static balance is considered to be altered when an individual cannot remain at least 30 seconds in each of the six conditions [28]. 
Improvement in the test was defined as the capacity to complete the test during the final evaluation when unable to complete the same in the initial evaluation.

\section{Functional mobility}

Functional mobility was evaluated by the Timed "Up \& Go" Test (TUGT) [29], which registers the time an individual takes to get up out of a chair, walk 3 meters, turn around, walk back and sit down again.

Elderly individuals without balance deficit are capable of completing the test in less than 10 seconds.

\section{Falls}

The number of falls in the year prior to the study [30] was solicited and noted in the initial evaluation and at the end of the trial (final evaluation). During the year of the study, patients in both groups received a diary and were orientated to write down the days that they fell.

At the end of the study, the difference in the number of falls/patient (final evaluation - initial evaluation) was compared between the Intervention Group and Control.

\section{Intervention}

The Balance Training Program consisted of 1 hour of exercises realized once a week, with a total of 40 classes, supervised by an experienced physiotherapist. This program was realized in a club (Associação Atlética Acadêmica Oswaldo Cruz - AAAOC) belonging to the Clinics Hospital, School of Medicine, University of São Paulo, located near to the Hospital. The balance exercises described by Tinetti and Suzuki $[3,11]$ were used. The type and mild to moderate intensity of the exercises used in the present study were chosen so that they could also be performed by elderly patients at home [3]. A list of weekly attendance controlled the absences of each patient.

Basic warm-up and stretching exercises

Prior to training, the patients participated in $15 \mathrm{~min}$ of warm-up and stretching exercises, consisting of head rotation, shoulder rotation and stretching of the upper and lower limbs. Walking was performed for $15 \mathrm{~min}$ with the supervision of a physiotherapist, who associated exercises for the upper limbs throughout the walk.

\section{Balance training}

Balance was realized in dynamic and static positions for a period of $30 \mathrm{~min}$. This consisted of walking in the tandem position (one foot in front of the other), walking on the tips of the toes and on the heel, walking sideways, walking while raising the leg and the contra-lateral arm, standing on one leg, and standing in the tandem position, while gradually increasing the period of permanence in these last two static positions [3, 31].

Home-based exercises

The patients were instructed and encouraged to continue the same exercises at home at least three times a week for $30 \mathrm{~min}$. A manual with instructions and illustrations for each exercise was distributed. The frequency of participation in the homebased exercises was noted each week by the physiotherapist.

\section{Data analysis}

Sixty-six consecutive patients were randomized in the present study. Data analysis was realized on 60 patients (30 Intervention Group and 30 Control), as six patients desisted (four Intervention Group and two Control). In the Intervention Group the reasons for desistance were: physical limitations as a result of falls $(n=1)$, foot pain $(n=1)$, personal reasons $(n=2)$; while in the Control group the reasons were: physical limitations as a result of falls $(n=1)$, personal reasons $(n=1)$.

The data were expressed as the mean and standard deviation (SD) for each variable and differences between the Intervention and Control groups were tested by the Student's $t$-test or Mann-Whitney test. The Chi-square test or Fisher's exact test was used to compare the number of patients in both groups (Intervention Group vs Control) for hypnotic and diuretic drug use, fracture history, CTSIB conditions (1-6) and improvement in CTSIB conditions. $P$ values $<0.05$ were considered significant.

\section{Results}

The basal characteristics of the patients of both groups were similar in relation to age, body mass index (BMI), fracture history, osteoporosis treatment, diuretics and hypnotics/ antidepressants use and bone mineral density (BMD), with no statistically significant differences between the two groups (Table 1).

Similarly, in the first evaluation, no differences occurred when comparing the Intervention Group and the Control Group in reference to: Berg Balance Scale (BBS) score, the number of patients that could not complete the Clinical Test of Sensory Interaction for Balance (CTSIB) in the six sensory conditions, the Timed "Up \& Go" Test (TUGT), and the number of falls/patients in the preceding year $(p>0.05)$ (Table 2). 
Table 1 Data at the onset of the study in relation to anthropometric parameters, fracture history, medication use and bone mineral density values (T-score) in the Intervention and Control groups

\begin{tabular}{llll}
\hline Variable & $\begin{array}{l}\text { Intervention } \\
(n=30)\end{array}$ & $\begin{array}{l}\text { Control } \\
(n=30)\end{array}$ & $p$-value \\
\hline Age, years & $74.57 \pm 4.82$ & $73.40 \pm 4.61$ & $0.342^{*}$ \\
BMI, kg/m & $24.39 \pm 4.49$ & $26.51 \pm 5.32$ & $0.100^{*}$ \\
Fracture history, $n(\%)$ & $13(43.3)$ & $16(53.3)$ & $0.438^{* * *}$ \\
Medication used for OP, $n$ & $2.37 \pm 1.50$ & $2.30 \pm 0.88$ & $0.498^{* *}$ \\
Hypnotics/ & $6(20.0)$ & $7(23.3)$ & $0.754^{* * *}$ \\
$\quad$ Antidepressants, $n(\%)$ & & & \\
Diuretics, $n(\%)$ & $14(46.7)$ & $16(53.3)$ & $0.606^{* * *}$ \\
Lumbar spine, T-score & $-2.83 \pm 1.07$ & $-2.62 \pm 1.12$ & $0.470^{* *}$ \\
Femur neck, T-score & $-2.70 \pm 0.75$ & $-2.75 \pm 0.90$ & $0.821^{* *}$ \\
Total femur, T-score & $-2.10 \pm 1.26$ & $-2.10 \pm 1.09$ & $0.990^{* *}$ \\
\hline
\end{tabular}

Data expressed in means \pm SD or percentage

BMI: body mass index, OP: Osteoporosis

*Student's $t$-test

**Mann-Whitney test

$* * *$ Chi-square test

\section{Adherence rate}

A high level of adherence was observed. Sixty percent of the patients participated in all of the exercise sessions at the club and absences occurred with the following justifications: doctor's appointment, the realization of laboratory exams or for personal reasons. In relation to home-based exercise, $76.67 \%$ of the patients realized exercises at least

Table 2 Data at the onset of the study for: Berg Balance Scale (BBS) score, number of patients that could not complete the Clinical Test of Sensory Interaction for Balance (CTSIB: condition 1: eyes open and firm surface; condition 2: eyes closed and firm surface; condition 3: eyes open, visual conflict and firm surface; condition 4: eyes open and unstable surface; condition 5: eyes closed and unstable surface; condition 6: eyes open, visual conflict and unstable surface), Timed "Up \& Go" Test (TUGT), and number of falls/patient in the preceding year in Intervention Group and Control

\begin{tabular}{llll}
\hline & $\begin{array}{l}\text { Intervention } \\
(n=30)\end{array}$ & $\begin{array}{l}\text { Control } \\
(n=30)\end{array}$ & $p$ value \\
\hline BBS, score & $48.80 \pm 4.10$ & $48.13 \pm 5.36$ & $0.900^{*}$ \\
CTSIB condition 1, $n(\%)$ & $0(0.0)$ & $1(3.3)$ & $1.000^{* *}$ \\
CTSIB condition 2, $n(\%)$ & $2(6.7)$ & $1(3.3)$ & $1.000^{* *}$ \\
CTSIB condition 3, $n(\%)$ & $2(6.7)$ & $2(6.7)$ & $1.000^{* *}$ \\
CTSIB condition 4, $n(\%)$ & $4(13.3)$ & $4(13.3)$ & $1.000^{* *}$ \\
CTSIB condition 5, $n(\%)$ & $15(50.0)$ & $12(40.0)$ & $0.604^{* *}$ \\
CTSIB condition 6, $n(\%)$ & $12(40.0)$ & $9(30.0)$ & $0.589^{* *}$ \\
TUGT, seconds & $14.31 \pm 4.03$ & $13.86 \pm 3.43$ & $0.610^{*}$ \\
Falls/patient preceding year, $n$ & $1.20 \pm 1.88$ & $0.87 \pm 0.86$ & $0.745^{*}$ \\
\hline
\end{tabular}

Data expressed in means $\pm \mathrm{SD}$ or percentage.

*Mann-Whitney test

**Fisher's exact test once a week, $40 \%$ of the patients exercised every day and $36.67 \%$ from one to four times a week.

Comparison between the Intervention Group and Control

The difference in BBS score (final-initial evaluation) was greater in the group which suffered intervention $(5.5 \pm 5.67$ vs $-0.5 \pm 4.88, p<0.001$ ) (Table 3 ).

Similarly, the percentage of patients in the Intervention group whose static balance improved in two sensory conditions (CTSIB, condition 5: eyes closed, unstable surface; and condition 6: eyes open, visual conflict, unstable surface) was statistically significant when compared to Control (CTSIB condition 5: 13 patients vs 1, $p<0.001$; CTSIB condition 6: 12 patients vs $1, p<0.001$ ) (Table 3).

Equally, a significant difference in the functional mobility, as measured by the TUGT (final-initial evaluation) was observed in the Intervention Group compared to Control $(-3.65 \pm 3.61$ vs $+2.27 \pm 7.18, p<0.001)$ (Table 3).

Parallel to these improvements in functional and static evaluation, a reduction in the number of falls/patient (finalinitial evaluation) was observed in the Intervention Group compared to Control $(-0.77 \pm 1.76$ vs $+0.03 \pm 0.98, p=$ 0.018) (Table 3).

\section{Discussion}

Few studies have been developed regarding balance training in patients with osteoporosis. The present longitudinal prospective study demonstrated that a program of balance training realized over a period of 12 months was effective in improving the functional and static balance, mobility and diminishing the number of falls in elderly women with osteoporosis.

Table 3 Differences (final evaluation - initial evaluation) in: Balance Berg Scale (BBS) score, number of patients showing improvement in Clinical Test of Sensory Interaction for Balance (CTSIB condition 5: eyes closed and unstable surface; condition 6: eyes open, visual conflict and unstable surface), time of Timed "Up \& Go" Test (TUGT), and number of falls/patient in the Intervention Group and Control

\begin{tabular}{llll}
\hline & $\begin{array}{l}\text { Intervention } \\
(n=30)\end{array}$ & $\begin{array}{l}\text { Control } \\
(n=30)\end{array}$ & $p$-value \\
\hline Difference BBS, score & $5.5 \pm 5.67$ & $-0.5 \pm 4.88$ & $<0.001^{*}$ \\
CTSIB condition 5, $n(\%)$ & $13(43.3)$ & $1(3.3)$ & $<0.001^{* *}$ \\
CTSIB condition 6, $n(\%)$ & $12(40.0)$ & $1(3.3)$ & $0.001^{* *}$ \\
Difference TUGT, seconds & $-3.65 \pm 3.61$ & $+2.27 \pm 7.18$ & $<0.001^{*}$ \\
Difference of falls/patient, $n$ & $-0.77 \pm 1.76$ & $+0.03 \pm 0.96$ & $0.018^{*}$ \\
\hline
\end{tabular}

Data expressed in means \pm SD or percentage.

*Mann-Whitney test

**Chi-square test 
In the present work, improvement in relation to functional balance was demonstrated by an increase in the BBS score in the final evaluation in the group submitted to the balance training program (Intervention Group). Similar results were shown in a study by Melzer et al. [32], where the patients who participated in the balance training obtained 64\% improvement in 3 months. The authors showed that the group who underwent the balance training demonstrated better performance than the group submitted to muscular strength training.

Programs that emphasize balance training are more effective at improving balance than those that consist primarily of aerobic, muscular strength or flexibility exercises [33].

Although it is a complex issue to evaluate the effectiveness of different types of exercises [34], balance training has shown beneficial results, with diminished risks of falls [35].

Another positive result of the present study was the improvement in the two difficult conditions of the Clinical Test of Sensory Interaction for Balance (condition 5: eyes closed, unstable surface, and condition 6: eyes open, visual conflict, unstable surface) in almost half the patients. Carter et al. [24] obtained an improvement in static balance using muscular strength training rather than balance training, though only in $6.3 \%$ of the patients. Thus, the present findings suggest that balance training leads to more evident positive results in static balance than does muscular strength training.

In relation to functional mobility, improvement was demonstrated by a reduction in the TUGT time in the patients submitted to intervention. These results are relevant, since research shows that compromised mobility increases the risk of dependency three- to five-fold, in activities of daily life [36]. This is not surprising, considering that mobility is an important component of daily life activities, for example: going to the shopping mall, to the supermarket, to the doctor or the cinema. Increased dependency could lead to institutionalization and diminished quality of life. Good balance is considered fundamental for improving mobility and preventing falls [36].

In parallel to the improvement in functional evaluation, an important reduction in the frequency of falls was observed. Although the effect of exercise in the prevention of falls in the elderly is yet to be proved, some studies show that physical activity reduces the risk by $40 \%$ [37]. On the other hand, in a consensus on prevention of falls in the elderly, the only exercises recommended to prevent falls are those which specifically target balance training [38].

Many studies that show improvement in balance and mobility present similar characteristics in their exercises. This suggests that the content and intensity of the exercise program could be more important than other intervention variables [39]. Moreover, differences in the administration of the exercise program, the professional experience of those who apply the exercises, the location in which the sessions are held and whether the exercise is conducted in group or realized individually, are all fundamental parameters which influence the success of the exercise program [39].

An important factor for the success of the exercise program is adherence. Contrary to the study by Forcan et al. [40], who stated that adherence to exercise in the elderly is weak, in the present study good adherence was observed, with more than half the patients present at all sessions. The current findings are similar to other studies which showed adherence up to $97.5 \%$ [17].

The success in adherence in the present study is probably due to the location where the exercises were performed (a pleasant, natural environment), to social interaction, and to the supervision of a physiotherapist. A secure environment, session supervision and the opportunity for social interaction reduce the feeling of isolation. A social support system is considered important in group activities, and helps sustain adherence and the effectiveness of the weekly exercise sessions and also the adherence to home-based exercises [35].

Similarly to our study, Robitaille et al. showed that an exercise program performed in groups improves the balance of the elderly in the community [41].

Another relevant factor was the use of a manual of instructions and illustrations for the realization of homebased exercises, which contributed to the continuity and adherence of the exercises performed. Each exercise was appropriately prescribed and illustrated by a physiotherapist, giving the patient adequate support. Descriptive and illustrated pamphlets have been used in some studies, complemented by a home-based exercise program, with positive results $[3,42]$.

The present positive results could also be related to the state of health of these patients, which was good in our patients. Buchner et al. [43] showed that exercise can have beneficial effects on health and on the risk of falls in certain subgroups of the elderly.

The physical and psychological benefits of the regularity of the sessions and the environment should never be ignored. Stimulating strategies and demonstrating ability in the transference from one exercise to another, consequently, maintaining enthusiasm during the exercises, can be practiced safely in groups [35].

Although our study did not use laboratory equipment [44] to substantiate the results obtained, we showed by reliable and reproducible scales and tests $[26,28,29]$ that balance training performed once a week, supervised by a physiotherapist, and complemented by home-based exercises, is very effective in the improvement of functional and 
static balance and mobility, and in the reduction of falls in elderly women with osteoporosis.

Acknowledgements Associação Atlética Acadêmica Oswaldo Cruz (AAAOC); Centro de Estudos em Reumatologia de São Paulo; CAPES (Coordenação de Aperfeiçoamento de Pessoal de Nível Superior).

\section{References}

1. Kuczynski M, Ostrowska B (2006) Understanding falls in osteoporosis: the viscoelastic modeling perspective. Gait Posture 23:51-58

2. Sinaki M, Brey RH, Hughes CA, Larson DR, Kaufman KR (2005) Balance disorder and increased risk of falls in osteoporosis and kyphosis: significance of kyphotic posture and muscle strength. Osteoporos Int 16:1004-1010

3. Suzuki T, Kim H, Yoshida H, Ishizaki T (2004) Randomized controlled trial of exercise intervention for the prevention of falls in community-dwelling elderly Japanese women. J Bone Miner Metab 22:602-611

4. Barraff LJ, Della PR, Williams N et al (1997) Practice guideline for the ED management of falls in community-dwelling elderly persons. Ann Emerg Med 30:480-492

5. Ashley MJ, Gryfe CI, Amies A (1977) A longitudinal study of falls in an elderly population, II: some circumstances of falling. Age Ageing 6:211-220

6. Silsupadol P, Siu KC, Shumway-Cook A, Woollacott MH (2006) Training balance under single and dual-task conditions in older adults with balance impairment. Phys Ther 86:269-281

7. Campbell AJ, Robertson MC, Gardner MM, Norton RN, Buchner DM (1999) Psychotropic medicine withdrawal and a home-based exercise program to prevent falls: a randomized controlled trial. J Am Geriatr Soc 47:850-853

8. Overstall PW, Exton-Smith AN, Imms FJ, Johnson AL (1977) Falls in the elderly related postural imbalance. Br Med J 1:261-264

9. Nelson RC, Amin MA (1990) Falls in elderly. Emerg Med Clin North Am 8:309-324

10. Campbell AJ, Borrie MJ, Spears GF et al (1990) Circumstances and consequences of falls experienced by a community population 70 years and over during a prospective study. Age Ageing 19:136-141

11. Tinetti ME, Baker, DI, McAvay G et al (1994) A multifactorial intervention to reduce the risk of falling among elderly people living in the community. N Engl J Med 331:821-827

12. Duncan PW, Studenski S, Chandler J, Prescott B (1992) Functional reach: predictive validity in a sample of elderly male veterans. J Gerontol 47:93-98

13. Hindmarsh JJ, Estes EH Jr (1989) Falls in older persons. Causes and interventions. Arch Intern Med 149:2217-2222

14. Horak FB, Shupert CL, Mirka A (1989) A components of dyscontrol in the elderly: A review. Neurobiol Aging 10:727-738

15. Close JCT, Glucksman E (2000) Falls in elderly: What can be done? Med J Aust 173:176-177

16. Steadman J, Donaldson N, Kalra L (2003) A randomized controlled trial of an enhanced balance training program to improve mobility and reduce falls in elderly patients. J Am Geriatric Soc 51:847-852

17. Sihvonen S, Sipilä S, Taskinen S, Era P (2004) Fall incidence in frail older-women after individualized visual feedback-based balance training. Gerontology 50:411-416

18. Nowalk MP, Prendergast JM, Bayles CM, D'amico FJ, Colvin GC (2001) A randomized trial of exercises programs among older individuals living in two long-term care facilities: The Falls-FREE Program. J Am Geriatr Soc 49:859-865
19. Judge J, Whipple RH, Wolfson LI et al (1994) Effects of balance exercises on isokinetic strength in older persons. J Am Geriatr Soc 42:937-946

20. Nitz JC, Choy NL (2004) The efficacy of a specific balancestrategy training programme for preventing falls among older people: a pilot randomised controlled trial. Age Ageing 33:52-58

21. Seidler RD, Martin PE (1997) The effects of short term balance training on the postural control of older adults. Gait Posture 6:224-236

22. Carter ND, Khan KM, Petit MA, Heinonen A, Waterman C, Donaldson MG, Janssen PA, Mallison A, Riddell L, Kruse K, Prior JC, Flicker L, McKay HA (2001) Results of a 10 week community based strength and balance training programme to reduce fall risk factors: a randomized controlled trial in 65-75 year old women with osteoporosis. Br J Sports Med 35:348-351

23. WHO World Health Organization (1994) Assessment of osteoporotic fracture risk and its role in screening for menopausal osteoporosis; WHO Technical Report Series, Geneva

24. Carter ND, Khan KM, McKay HA, Petit MA, Waterman C, Heinonen A et al (2002) Community-based exercise program reduces risk factors for falls in 65- to 75-year-old women with osteoporosis: randomized controlled trial. CMAJ 167:997-1004. Erratum in: CMAJ 2003 168:152

25. American College of Sports Medicine (1995) Guidelines for Exercise Testing and Prescription, 5th Ed. Baltimore: Williams and Wilkins, pp. 1-373

26. Miyamoto ST, Lombardi Junior I, Berg KO, Ramos LR, Natour J (2004) Brazilian version of the Berg balance scale. Braz J Med Biol Res 37:1411-1421

27. Whitney SL, Poole JL, Cass SP (1998) A review of balance instruments for older adults. Am J Occup Ther 52:666-671

28. Shumway-Cook A, Horak FB (1986) Assessing the influence of sensory interaction on balance, suggestion from the field. Phys Ther 66:1548-1550

29. Podsiadlo D, Richardson S (1991) The Timed "Up \& Go": a test of basic functional mobility for frail elderly persons. JAGS 39:142-148

30. Tinetti ME, Speechley M, Ginter SF (1988) Risk factors for falls among elderly persons living in the community. N Engl J Med 319:1701-1707

31. Van Norman KA (1995) Exercise programming for older adults. Human Kinetics, Champaign, IL, USA, pp 41-66

32. Melzer I, Benjuya N, Kaplanski J (2005) Effect of physical training on postural control of elderly. Harefuah 114:839-844

33. Rogers ME, Fernandez JE, Bohlken RM (2001) Training to reduce postural sway and increase functional reach in the elderly. J Occup Rehabil 11:291-298

34. Chang JT, Morton SC, Rubenstein LZ, Mojica WA, Maglione M, Suttorp MJ, Roth EA, Shekelle PG (2004) Interventions for the prevention of falls in older adults: systematic review and metaanalysis of randomised clinical trials. BMJ 328:680-683

35. Skelton DA (2001) Effects of physical activity on postural stability Age Ageing 30:33-39

36. Frank JS, Patla AE (2003) Balance and mobility challenges in older adults: Implications for preserving community mobility. Am J Prev Med 25:157-163

37. Barnett A, Smith B, Lord SR, Williams M, Baumand A (2003) Community-based group exercise improves balance and re duces falls in at-risk older people: a randomised controlled trial. Age Ageing 32:407-414

38. Feder G, Cryer C, Donovan S (1998) Guidelines for the prevention of falls in older people. Queen Mary and Westfield College, London

39. Means KM, Rodell DE, O'Sullivan PS (2005) Balance, mobility, and falls among community-dwelling elderly persons: effects of a rehabilitation exercise program. Am J Phys Med Rehabil 84:238250 
40. Forcan R, Pumper B, Smyth N, Wirkkala H, Ciol MA, ShumwayCook A (2006) Exercise adherence following physical therapy intervention in older adults with impaired balance. Phys Ther 86:401-410

41. Robitaille Y, Laforest S, Fournier M, Gauvin L, Parisien M, Corriveau H, Trickey F, Damestoy N (2005) Moving forward in fall prevention: an intervention to improve balance among older adults in real-world settings. Am J Public Health 95:2049-2056

42. King MB, Whipple RH, Gruman CA, Judge JO (2002) The performance enhancement project: improving physical perfor- mance in older persons. Arch Phys Med Rehabil 83:10601069

43. Buchner DM, Cress ME, de Lateur BJ, Esselman PC, Margherita AJ, Price R, Wagner EH (1997) The effect of strength and endurance training of gait, balance, fall risk, and health services use in community-living older adults. J Gerontol A Biol Sci Med Sci 52:218-224

44. Sinaki M, Brey RH, Hughes CA, Larson DR, Kaufman KR (2005) Significant reduction in risk of falls and back pain in osteoporotickyphotic women through a spinal proprioceptive extension exercise dynamic (SPEED) program. Mayo Clinic Proceedings 80:849-855 